\title{
Macht - Leistung - Kultur: Staatenvergleiche vom 17. bis ins frühe 20. Jahrhundert
}

\author{
Willibald Steinmetz
}

Eingegangen: 9. September 2020 / Angenommen: 8. Oktober 2020 / Online publiziert: 18. Mai 2021 (C) Der/die Autor(en) 2021

Zusammenfassung Praktiken des Vergleichens und Bewertens von Staaten mittels Zahlen haben eine lange Geschichte. Seit der Frühen Neuzeit erlebten sie einen Aufschwung infolge fiskal-militärischer Rivalität zwischen größeren und kleineren Mächten innerhalb wie außerhalb Europas. Im Laufe des langen 19. Jahrhunderts kamen zusätzliche Kriterien ins Spiel: Neben militärischer und demografischer Stärke wurden nun ökonomische Leistungsfähigkeit, soziale Vorsorge für die Einwohner sowie kulturelle Errungenschaften wichtiger. In einem ersten Schritt benennt der Beitrag wesentliche Voraussetzungen für die heute etablierten Praktiken des Rankings von Staaten: Serialität und Standardisierung der Datenerhebung, Existenz international anerkannter centres of calculation, relevante Öffentlichkeiten als Nachfrager für Staatenvergleiche, eine Vision von Geschichte als Fortschritt, welche staatliche Akteure nötigte, sich im Verhältnis zu anderen als ,,vorauseilend“ oder ,zurückgeblieben“ einzuordnen. Die empirischen Teile des Beitrags widmen sich der Entfaltung staatenvergleichender Praktiken in West- und Mitteleuropa vom späten 17. bis ins frühe 20. Jahrhundert. In exemplarischen Studien werden Autoren und Institutionen behandelt, die Staaten quantifizierend zu vergleichen suchten (englische politische Arithmetiker, deutsche Tabellenstatistiker, nationale statistische Bureaus, internationale statistische Kongresse). Ebenfalls berücksichtigt werden qualitative Staatenvergleiche in der deskriptiven deutschen „Statistik“ des 18. Jahrhunderts oder durch visuelle Präsentationen auf den Weltausstellungen des 19. Jahrhunderts. Schließlich kommen im Beitrag auch Kritiker zu Wort, die im Namen der Singularität von Nationen, Staaten oder Imperien gegen deren Reduktion auf wenige zählbare Größen protestierten. Die Geschichte der Staatenvergleiche im 18. und 19. Jahrhundert lief also keineswegs geradlinig auf immer mehr Quantifizierung hinaus. Vielmehr war

\footnotetext{
W. Steinmetz $(\bowtie)$

Fakultät für Geschichtswissenschaft, Philosophie und Theologie, Universität Bielefeld

Universitätsstr. 25, Postfach 1001 31, 33501 Bielefeld, Deutschland

E-Mail: willibald.steinmetz@uni-bielefeld.de
} 
sie die Geschichte einer umstrittenen Praxis, in der ,progressive“ besser-schlechterVergleiche stets mit solchen Vergleichen rivalisierten, die auf essenzialisierte Andersartigkeit abhoben.

Schlüsselwörter Statistik · Fiskal-militärischer Staat · Wettbewerb · Progressiver Vergleich · Nationale Singularität

\section{Power, Performance, Culture: The Comparison of States From the 17th Century to the Early 20th Century}

Abstract Practices of comparing and grading states by numbers have a long history. Taking off in the early modern period, these practices were triggered by fiscal-military rivalry between major and minor powers within Europe and beyond. While military and demographic strength continued to be important yardsticks, additional criteria came to the fore in the 19th century: economic performance, social provisions for inhabitants, and cultural achievements. In a first step, the article enumerates the preconditions for today's elaborated state ranking practices: seriality and standardization of data-collection, the existence of internationally recognized centers of calculation, relevant publics creating a demand for state comparisons, and a progressive view of history that induces state actors to imagine themselves as being "ahead" or "lagging behind." The empirical parts of the article are devoted to the gradual unfolding of state-comparing practices within Western and Central Europe from the late 17 th century to the early 20 th century. Exemplary studies deal with authors and institutions that measured state powers in quantitative terms (English political arithmeticians, German tabular statisticians, national statistical bureaus, international statistical congresses). Attention is also paid to qualitative comparisons in 18th-century German Statistik and visual displays at 19th-century world fairs. The article also takes account of early 19th-century critics who, on behalf of each nation's singularity, protested against the reduction of states (or empires) to just a handful of numerically countable categories. The story of 18th- and 19th-century state comparisons is therefore not one of a linear development towards ever more quantification, but that of a contested practice that includes progressive better-worse comparisons as well as comparisons insisting on essentialized differences.

Keywords Statistics · Fiscal-military state $\cdot$ Competition $\cdot$ Progressive comparison $\cdot$ National singularity

\section{Einleitung}

Vergleichende Staatenbewertungen in Form von Rankings sind heute eine gängige Praxis. Für das Jahr 2014 hat man allein 95 Indices gezählt, in denen Staaten unter verschiedenen Gesichtspunkten in eine Rangordnung gebracht werden (Cooley und Snyder 2015, S. 3, S. 194-203). Historisch gesehen sind derartige Indices und ähnliche Formen des Bewertens und Vergleichens von Staaten extrem voraussetzungsreich. Im Folgenden werden zunächst von der Gegenwart ausgehend die 
bis in die Frühe Neuzeit zurückreichenden Voraussetzungen derartiger Praktiken benannt und ihren historischen Kontexten zugeordnet (Abschn. 2). Im Anschluss werden vom späten 17. Jahrhundert ausgehend einige Stationen der Entfaltung des Kategorisierens, Bewertens und Vergleichens von Staaten genauer beleuchtet. Dabei zeigt sich, dass „Handelsneid“ (Hont 2010) und mehr noch die zahlreichen Kriege vom späten 17. Jahrhundert bis zur napoleonischen Ära entscheidende Anstöße für die Fortentwicklung von Techniken des Messens, Zählens und vergleichenden Bewertens von Machtressourcen - „Staatskräften“ in der damaligen Terminologie lieferten (vgl. Speich Chassé 2020). Dass „Staaten“ ebenso wie verschiedene Klassen von Staaten (Großmächte, kleinere Staaten) sich überhaupt im Bewusstsein der Regierenden und Regierten als Vergleichseinheiten festsetzten, resultierte nicht zuletzt aus jenen Praktiken des Zählens, Messens und Bewertens von „Staatskräften“, die im 18. Jahrhundert aufkamen (Abschn. 3).

Im Laufe des in Europa relativ friedlichen 19. Jahrhunderts traten die industriell-technische Leistungsfähigkeit und die kulturell-moralische „Fortschrittlichkeit“ als Kriterien hinzu, ohne dass die machtbezogenen, leicht mess- und zählbaren Größen wie Fläche, Bevölkerungsstärke und-dichte, Finanzkraft und Militärmacht an Bedeutung verloren. Die kompetitiven Staatenvergleiche weiteten sich damit, so die im Titel angedeutete These, auf immer mehr Bereiche aus: von der Macht über die Leistung bis zur Kultur. Mit ihrer Ausweitung wuchs auch die Kritik an diesen Vergleichspraktiken. Im Namen des Einmaligen, Besonderen, qualitativ je Verschiedenen protestierten die Kritiker, ob romantisch, national oder historistisch inspiriert, gegen die gleichmachende Reduktion der Individuen und kollektiven Einheiten (Nationen, Gruppen) auf statistisch verwertbare Größen. Die trotz aller Kritik sich weiter vollziehende Perfektionierung der innerstaatlichen Statistiken zeitigte ihrerseits den paradoxen Effekt, dass aussagekräftige statistische Vergleiche zwischen den Staaten erschwert wurden. Seit der Mitte des 19. Jahrhunderts wurde zwar viel über gemeinsame Standards des Kategorisierens, Zählens und Messens diskutiert, doch konnte man sich bis zum Ersten Weltkrieg nur in Teilgebieten auf Standards einigen. Unmittelbar anschauliche, qualitative Vergleichsinszenierungen boten ein gewisses Substitut für unzureichende quantifizierende Staatenvergleiche, prominent auf den Weltausstellungen seit 1851 (Abschn. 4). Mit dem Völkerbund und seinen Unterorganisationen, vor allem der International Labour Organization (ILO), traten nach 1919 internationale Organisationen auf den Plan, die für das Methodenwissen, das heutigen Staatenrankings zugrunde liegt, elementare Bausteine lieferten. Doch kam es noch nicht zu Praktiken des Staatenrankings im heute geläufigen Sinne. Ein Ausblick auf diese Übergangsphase beschließt den Beitrag.

\section{Voraussetzungen für moderne Staatenrankings im Rückblick}

Von Einzelphänomenen abstrahierend lassen sich wenigstens fünf Voraussetzungen bestimmen, die erfüllt sein mussten, damit sich die heute bekannten Formen des Staatenrankings oder-ratings herausbilden konnten (vgl. Werron 2012, 2020). Diese Voraussetzungen werden nun von der Gegenwart aus zurückblickend in aller Kürze benannt. 


\subsection{Serielle Datenerfassung nach international gleichen Standards}

Staatenvergleiche in Form von Rankings setzen voraus, dass die jeweils relevanten Daten, etwa zur Handelsbilanz, zur Arbeitslosenquote oder zur Zahl der Intensivbetten in Krankenhäusern, in regelmäßigen Intervallen, also als Serie, und möglichst gleichzeitig sowie nach gleichen Standards erhoben werden. Nur so kann der relative Auf- oder Abstieg der Staaten über längere Zeit hinweg verfolgt werden, und nur so gewinnt das Ranking den ihm zugedachten Sinn, als Antrieb zu dienen für vermehrte Anstrengungen derer, die zurückgefallen sind, oder als Bestätigung herzuhalten für diejenigen, die in der Rangtabelle einen Sprung nach vorn gemacht haben. Auf einzelstaatlicher Ebene findet sich die serielle Erhebung einzelner Daten, vor allem zur Bevölkerung und landwirtschaftlichen Produktion, und ihre statistische Aufbereitung in West- und Mitteleuropa seit dem späten 18. Jahrhundert (Behrisch 2016a), in den meisten anderen Weltregionen nicht vor dem späten 19. Jahrhundert. Eine staatenübergreifende Serialisierung und Standardisierung der Datenerfassung wurde seit 1853 auf internationalen statistischen Kongressen und seit 1885 im Rahmen des International Statistical Institute als Postulat formuliert (Randeraad 2010), doch erst in den Jahrzehnten zwischen 1919 und 1949 zeitigten die Bemühungen bescheidene Erfolge. Bis heute ist dieses Problem alles andere als befriedigend gelöst (Heintz 2012). Gerade die frühe Regularisierung der Statistik auf nationaler Ebene in den größten und mächtigsten Staaten erschwerte die Standardbildung auf internationaler Ebene, denn die Maßstäbe, Kriterien und Methoden wurden selbst zum Gegenstand des Prestigewettstreits der Großmächte (Bemmann 2019a; Moses 2019; Speich Chassé 2019).

\subsection{Organisationen mit ausreichender Kompetenz und anerkannter Autorität}

Eine staatenübergreifende Datenerhebung verlangt erhebliche organisatorische, technische und personelle Kapazitäten sowie anerkannte Autoritäten, um die Einhaltung von Standards zu gewährleisten. Während in der Gegenwart zahlreiche privatwirtschaftlich oder moralpolitisch motivierte Nichtregierungsorganisationen dazu fähig sind, Staaten vergleichend unter qualitativen oder quantitativen Aspekten zu bewerten (oft durch Zweitverwertung öffentlich verfügbarer Daten), waren vor dem Zeitalter elektronischer Datenverarbeitung, von Ausnahmen wie den Credit Rating Agencies abgesehen, nur internationale oder suprastaatliche Organisationen (ILO, UNO, OECD) dazu in der Lage. Nichtstaatliche Expertengruppen führten zwar vereinzelt seit der Mitte des 19. Jahrhunderts, Einzelautoren sogar schon seit dem 18. Jahrhundert, Staatenvergleiche nach qualitativen oder quantitativen Kriterien durch, doch eine regelmäßige Praxis kam auf dieser Basis nicht zustande. Auch Organisationen wie die ILO oder die OECD waren (und sind) für ihre Staatenvergleiche auf die freiwillige Kooperation einzelstaatlicher Agenturen der Datenproduktion angewiesen. Sie verfügen bis heute nur über indirekte Druckmittel (shame and blame, Entzug von Hilfen oder Krediten), um auf die Einheitlichkeit der Kategorienbildung und die regelkonforme Anwendung der Zähl- und Messmethoden in den Einzelstaaten hinzuwirken. Letztlich sind Staatenrankings oder -ratings bis heute davon abhängig, dass die staatlichen Akteure, wie widerstrebend auch immer, mitwirken und die ge- 
wünschten Daten, wie geschönt auch immer, freigeben. Eine minimale Akzeptanz des Prinzips der Rankings und Ratings und der Autorität derer, die sie anstellen, ist erforderlich.

\subsection{Relevante und interessierte Dritte als Nachfrager und Adressaten}

Staatenrankings erfüllen nur dann ihren Zweck, wenn es ein Publikum, bestehend aus Experten, Massenmedien, Politikern oder Laien, gibt, das sich für die hierarchisierenden Bewertungen interessiert und als dritte Instanz fungiert, die den Wettbewerb der um Prestige ringenden Staaten in Schwung hält (Werron 2020). Die Bereitschaft, sich auf Rankings einzulassen, beruht wesentlich darauf, dass die verglichenen Einheiten, hier also Staaten und ihre Regierungen, vor den Augen eines solchen Publikums gut dastehen wollen. Wie zentral die Figur des beobachtenden, mitunter auch sich einmischenden „Dritten“ in der Staatenkonkurrenz ist, wird heute etwa bei den in gewissen Abständen neu aufgelegten Messungen der Schreibund Lesekompetenz von Schülerinnen und Schülern (PISA-Tests) offenkundig. In der Weltgesellschaft des späten 20. und frühen 21. Jahrhunderts ist das Publikum von Staatenvergleichen tendenziell global, anonym und sozial kaum eingrenzbar. In der zweiten Hälfte des 19. Jahrhunderts waren die wiederkehrenden Weltausstellungen mit den sie begleitenden Expertenkongressen eine Gelegenheit, bei der die Rangfolge der Staaten für ein millionenfach anwesendes oder nachlesendes Laienpublikum zum Thema gemacht wurde (Geppert 2013; Haltern 1973). Letztlich ist aber die Größe, räumliche Ausdehnung und soziale Breite des Publikums für das Funktionieren des Antriebs, der die Prestigekonkurrenz der Staaten in Gang hält, sekundär. Auch wenn die beobachtende dritte Instanz, wie überwiegend im 19. Jahrhundert, nur aus bürgerlichen Lesepublika und fachspezifischen Expertengruppen oder, wie im 18. Jahrhundert, nur aus europaweit vernetzten Gelehrtenund adligen Hofgesellschaften bestand, stellte sich der Effekt des Wettbewerbs ein. $\mathrm{Zu}$ unterbinden gewesen wäre er nur durch rigorose Geheimhaltung der Staatenvergleiche und der ihnen zugrundeliegenden Daten. Tatsächlich waren europäische Monarchen und regierende Bürokraten bis ins frühe 19. Jahrhundert bestrebt (wenn auch selten erfolgreich darin), sensible Daten zu Staatseinkünften, Staatsschulden, Militärmacht und anderen Ressourcen nach Möglichkeit unter Verschluss zu halten und so die Publikation statistisch basierter Staatenvergleiche zu erschweren (Klueting 1986; Göderle 2018). Der Streit um die Publizität statistischer Daten zu den „Staatskräften“ wurde in den Jahrzehnten um 1800 selbst zum öffentlichen Thema (etwa bei Schlözer 1804).

\subsection{Staatenwettbewerb als Denkmodell}

Ein auf Staaten bezogenes Prestigedenken, das sich an der Fort- oder Rückschrittlichkeit im Vergleich zu anderen bemisst, setzt voraus, dass staatliche Akteure überhaupt der Vorstellung anhängen, sie befänden sich in einem auf Steigerung angelegten Wettbewerb des Einholen-, Mithalten- oder gar Überholen-Wollens. Diese Denkvoraussetzung wird bei der Betrachtung gegenwärtiger Staatenrankings leicht übersehen, denn sie liegt auf der Ebene fundamentaler, in der Moderne selten ange- 
zweifelter Zeit- und Geschichtsvorstellungen. Diese wandelten sich, Reinhart Koselleck zufolge, seit dem späten 18. Jahrhundert in dem Sinne, dass „Geschichte“ nun erst von aufklärerischen Geschichtsschreibern und idealistischen Philosophen als ein linear fortschreitender, zukunftsoffener Prozess verstanden wurde (Koselleck 1979). Die Verbreitung dieser Sichtweise in Europa, ablesbar an neuen Bewegungsbegriffen im Kollektivsingular wie „Geschichte“, „Entwicklung“, ,Zivilisation“ oder „Fortschritt“, sorgte im 19. Jahrhundert dafür, dass sich Staaten ebenso wie andere Handlungseinheiten mehr und mehr genötigt fühlten, sich selbst und die jeweiligen Rivalen auf einer Skala schon erreichter oder noch zu erreichender, andernorts bereits verwirklichter oder zukünftig denkbarer Fortschritte relationierend zu verorten. Für die in der Selbstwahrnehmung Schwächeren, im späteren 19. Jahrhundert beispielsweise Staaten wie Japan oder China, hieß das zunächst, dass sie von den neuerdings Stärkeren lernen wollten, um ihnen als unabhängiger Akteur überhaupt widerstehen zu können. Geschichtsphilosophische Entwürfe waren mithin, so wird hier argumentiert, nicht unbedingt erforderlich, um den Handlungsmodus des ,progressiven Vergleichens“ in Gang zu setzen und aufrechtzuerhalten. Vielmehr findet man diesen Modus des Vergleichens schon vor und jenseits seiner reflexiven Verwendung angelegt in den Praktiken und Überlegungen derer, die zahlenbasierte oder qualitative Staatenvergleiche anstellten. Man kann sogar weitergehen und argumentieren, dass der Wettbewerb der großen europäischen Staaten um Handelsvorteile sowie die Notwendigkeit, die eigene Fähigkeit zur Kriegsführung (stehende Heere, Flotten, Fiskalstärke, Bevölkerungszahl) mit derjenigen der Rivalen abzugleichen, selbst ein treibendes Moment bei der Herausbildung eben jener Zeit- und Geschichtswahrnehmung als „Fortschritt“ gewesen ist (vgl. für England Slack 2015).

\section{5 „Staaten“ als Einheiten für kompetitive Vergleiche}

Ebenso wie der Gedanke des Staatenwettbewerbs ist auch die Kategorie „Staat“ - historisch gesehen - keineswegs selbstverständlich. Zu betonen ist, dass hier bewusst von „Staaten“, nicht von „Nationalstaaten“" gesprochen wird. Es ist in der neueren historischen Forschung mittlerweile Konsens, dass der territorial klar begrenzte, nach innen souveräne, von außen als solcher anerkannte und auf einem ethnisch, kulturell oder politisch definierten Zugehörigkeitsgefühl der Einwohner beruhende „Nationalstaat“ bis ins frühe 20. Jahrhundert hinein nicht die allseits geteilte Norm der Selbstorganisation politischer Handlungseinheiten gewesen ist, für die er lange gehalten worden ist (vgl. nur Osterhammel 2009, S. 565-678; Leonhard und von Hirschhausen 2009). Sogar für die angebliche Hochphase des Nationalstaats vom späten 19. Jahrhundert bis in unsere Gegenwart wird nunmehr die Vielfalt der Formen von Staatlichkeit stärker herausgestellt. Für die erste Hälfte des 19. Jahrhunderts und die Frühe Neuzeit (sowie in globaler Perspektive) kann vom Nationalstaat als dominantem Modell ohnehin nicht die Rede sein. Das Modell des Nationalstaats konkurrierte mindestens bis zur Phase der rapiden Dekolonisation (ab 1945) mit dem multiethnischen oder rassistisch-hierarchischen Imperium als Alternativmodell politischer Organisation, ebenso aber auch mit föderalen Ordnungen sowie mit Formen abgestufter Staatlichkeit innerhalb größerer Einheiten (Kolonien, Dominions im Britischen Empire, Protektorate, Gebiete mit Autonomiestatus). 
Für die letztgenannten Formen - außerhalb Europas wie innerhalb - galt allerdings seit dem späten 19. Jahrhundert in der Regel die volle innere und äußere Souveränität als Maßstab und Zielpunkt der Selbstbeurteilung. Die Staatenvergleiche vom 18. bis zum 20. Jahrhundert konnten sich auf die verschiedenen genannten Formen der Staatlichkeit vom Imperium bis zum autonomen Gebiet erstrecken, was die Vergleichbarkeit nicht erleichterte. Dabei wurden die Staatsgebilde ihrerseits oft nach Größe und Form der Staatlichkeit kategorisiert und in eine Rangfolge, mit oder ohne Temporalkoeffizient, gebracht. Gleiches galt auch noch in der Zwischenkriegszeit, etwa für die Mandatsgebiete des Völkerbunds (Pedersen 2017; Wheatley 2017a, b). Und auch in der Gegenwart gibt es eigene Staatenrankings oder -ratings, die mit Kategorien wie „fragile states“, „failed states“ operieren, um Rangordnungen von „stateness“ herzustellen (Bhuta 2015).

\subsection{Exkurs: Staaten- und Nationenvergleiche}

Von den in diesem Aufsatz behandelten Staatenvergleichen zu unterscheiden sind Nationenvergleiche, die schon im europäischen Spätmittelalter fassbar werden, etwa auf dem Konstanzer Konzil (1414-18) oder bei den deutschsprachigen Humanisten (vgl. dazu Hirschi 2005, allerdings mit begrifflichen Unschärfen). Die nationes des Spätmittelalters waren weder deckungsgleich mit einzelnen Ethnien oder Sprachgruppen noch gar mit den dynastisch definierten Herrschaftsverbänden oder anderen Formen politischer Obrigkeit (Stadtrepubliken). Wenn überhaupt, näherte sich die französische Monarchie nach dem Ende des Hundertjährigen Kriegs und der Niederwerfung Burgunds am ehesten einem Zustand der Deckungsgleichheit von „Nation“, Ethnie, Sprache und Staat an. In den „Nationen“-Vergleichen des 15. und 16. Jahrhunderts finden sich neben topoiartigen Völkerstereotypen bereits Formen einer kompetitiven Gegenüberstellung. Dieser Wettbewerb bezog sich überwiegend auf die Anciennität des Vorrangs in einer mehr oder weniger imaginären Vergangenheit. Der Blick richtete sich aber teilweise schon auf qualitative, manchmal auch quantifizierbare Gegebenheiten der Gegenwart, vereinzelt sogar auf zukünftige Potenziale der „Nationen“. Der Form nach gehören die spätmittelalterlich-humanistischen ,Nationen"-Vergleiche somit in die Vorgeschichte der modernen Staatenvergleiche, dem Objekt nach sind sie davon zu unterscheiden.

\section{Der Vergleich der ,Staatskräfte“ im langen 18. Jahrhundert (1680-1815)}

Nach den vorstehenden Überlegungen scheint es sinnvoll, den nun beginnenden vorwärts schreitenden Erkundungsgang im späten 17. Jahrhundert einsetzen zu lassen, also in einer Epoche sich verstärkender kommerzieller und militärischer Rivalität der großen maritimen Kolonialreiche und europäischen Kontinentalmächte. Dies war zugleich eine Epoche, in der man begann, die Interessen der Herrscherdynastien zumindest begrifflich von den Interessen der Staaten abzukoppeln (,Staatsräson“). Seit dem beginnenden 18. Jahrhundert kam ein Denken in Kategorien des „Gleichgewichts“ der Mächte hinzu. Das Bild des „Gleichgewichts“ war ein zusätzlicher 
Ansporn für machtbezogene Kräftevergleiche, die bis in die Ära des Wiener Kongresses anhielten. Die im 18. Jahrhundert lange vorherrschende Wirtschaftsdoktrin des Merkantilismus, ähnlich als deutsche Variante der Kameralismus, beförderte ebenfalls ein Denken in den Kategorien des kompetitiven, ressourcenberechnenden Staatenvergleichs, bei dem neben der „Glückseligkeit“ der Untertanen (Behrisch 2016a) letztlich der fiskal-militärische Gesichtspunkt ausschlaggebend war, vor allem bei den Großmächten (Rommelse und Downing 2014; Reinert 2014). Der tendenziell weltweite Wettbewerb zwischen den Staaten blieb auch in der neueren liberalen Doktrin des Freihandels die maßgebliche Idee, allerdings mit dem Unterschied, dass nun nicht mehr militärische Machtsteigerung, sondern friedliche, ökonomisch-technische Leistungsverbesserung, also der „Wohlstand der Nationen“ (Adam Smith), das Ziel der Konkurrenz sein sollte (Hont 2010). Für qualitative und - zunehmend - auch quantifizierende Staatenvergleiche bestand vor dem skizzierten politischen und ideologischen Hintergrund ein stetig wachsendes Interesse, sowohl auf Seiten der Regierenden als auch, gegen Ende des 18. Jahrhunderts wirksamer, bei einem ,patriotisch“ gesinnten gebildeten Publikum. Auf dieses Interesse antworteten die anfangs vorwiegend beschreibende, nur gelegentlich zahlengestützte „Statistik“ (Staatenkunde) sowie - zuerst in England - die ,politische Arithmetik“.

3.1 Wissen gewinnen über den Staat: „Staatstafeln“ und ,politische Arithmetik“ um 1680

Nahezu zeitgleich begannen in den Jahrzehnten um 1680 hohe Funktionsträger, Gelehrte im Staatsdienst und Privatgelehrte in verschiedenen west- und mitteleuropäischen Staaten über Mittel und Wege nachzusinnen, wie Regierende systematischer als zuvor genaue Kenntnisse über die Einwohner und Ressourcen des eigenen Landes erlangen könnten. Bei dem Philosophen und (seit 1676) braunschweigischlüneburgischen Hofbibliothekar Gottfried Wilhelm Leibniz (1646-1716) erschienen diese Vorschläge eingebettet in ein enzyklopädisches Programm zur Akkumulation und Reorganisation des gesamten Weltwissens, das sich in Akademie- und Archivgründungen, Naturalienkammern, Bilderatlanten und ähnlichen Sammlungskonzepten realisieren sollte (Segelken 2010, S. 49-56). In seinem an Herzog Ernst August gerichteten Entwurff gewisser Staats-Tafeln (1680) stellte Leibniz das Ideal eines Regenten vor, der dank wohlgeordneter Tabellen in die Lage versetzt werde, ,Alles ... nicht allein zu finden, sondern auch was zusammen gehöret, gleichsam in einen augenblick zu übersehen“. Um an die „nöthigen Nachrichtungen zu gelangen“, solle man sich „der bereits habenden scripturen“ bedienen, außerdem „erfahrene Leüte“ vernehmen und, wo beides nicht genüge, ,,zu der inqvisition und besichtigung selbsten“" schreiten (Leibniz [1680a] 1986a, S. 345, 347). Mit der einmaligen Informationssammlung sei es indes nicht getan. Der ideale Regent sollte vergleichen können. Und so plädierte Leibniz ebenfalls 1680 für die „Bestellung eines Registratur-Amts“, dank dessen man die ,zerstreüte nachrichtungen ... gegen einander halten“ und so „die comparation unterschiedener lande, orthe unter einer Herrschafft, und unterschiedener zeiten eines Landes gegen einander" vollziehen könne. Der Nutzen einer solchen räumliche und temporale Vergleiche ermöglichenden Registratur bestehe darin, ,allerhand reale Verbeßerungen“ anzuregen, ,die nicht ex theoria, sondern 
media locorum aliorum praxi genommen, also sicher und vermittelst solcher leüte so damit umbgangen, auch anderswo, so viel thunlich anzubringen wären“ (Leibniz [1680b] 1986b, S. 377 f.). Derlei administrative Verbesserungen durch Imitation von andernorts verwirklichten best practices sollten sich übrigens, so Leibniz weiter, auch auf das Registraturwesen selber erstrecken.

Um nützliche Verbesserungen mithilfe tabellarisch systematisierten Wissens ging es wenig später auch dem französischen General und Festungsbaumeister Ludwigs XIV., Sébastien Le Prestre de Vauban (1633-1707). Vauban dürfte Leibniz, der seit 1675 ausländisches Mitglied der Académie Royale des Sciences war und sich häufig in Frankreich aufhielt, gekannt haben. Ähnlich wie bei Leibniz findet sich auch in Vaubans 1686 publizierter Schrift Méthode generalle et facile pour faire le dénombrement des Peuples die Vision, dass der Herrscher in einem „Augenblick“ alle Kräfte seines Staatswesens vor sich haben solle. Es müsse ihm, so Vauban, eine Befriedigung sein, ,innerhalb einer Stunde von seinem Kabinett aus den gegenwärtigen und vergangenen Zustand eines großen Königreichs, dessen Haupt er ist, selbst durchlaufen zu können und mit Sicherheit wissen zu können, worin seine Größe, seine Reichtümer und seine Kräfte bestehen“ (Vauban [1686, S. 14], Vilquin 1975, S. 256, meine Übers.; vgl. auch Damien 2003). Die von Vauban anvisierten, lokal zu erhebenden, von den Intendanten und Provinzgouverneuren zu kollationierenden tabellarischen Übersichten sollten neben der Bevölkerungszahl (differenziert nach Geschlecht, Familienstand, sozialem Stand und Beschäftigung) auch den Viehbestand, landwirtschaftliche Erträge und - perspektivisch - die mögliche Steuerleistung sowie auch die Fähigkeit von Regionen, Truppenkontingente zu unterhalten, erfassen und darstellen. Diese Tabellen seien jährlich zu aktualisieren, sodass ,der Vergleich der alten mit den neuen Zählungen“ den Herrscher in die Lage versetze, notwendige ,Verbesserungen“ (reparations) in den Provinzen aufgrund größerer Sachkenntnis zielgenauer anordnen zu können (Vauban [1686, S. 15], Vilquin 1975, S. 256). Für den französischen General Vauban ging es mithin ähnlich wie für den hannoveraner Hofbibliothekar Leibniz um Verbesserungen, die der Fürst in seinem eigenen Lande durchsetzen sollte, doch hatten Vaubans Vorschläge darüber hinaus deutlicher als bei Leibniz eine außenpolitische, fiskal-militärische Dimension. Vaubans Zählungen und Tabellen sollten die relative Stärke Frankreichs und das Prestige seines Königs sichtbar werden lassen. Hauptmaßstab für letzteres war für Vauban allerdings, anders als für seinen Herrn, Ludwig XIV., nicht der Ruhm siegreich gewonnener Schlachten, sondern die „Erhaltung und Vermehrung“ der Zahl seiner Untertanen: ,QUE LA GRANDEUR DES ROIS SE MESURE PAR LE NOMBRE DE SES SUJETS“ schrieb Vauban in kapitalen Lettern als erste „Maxime“ dem König ins Stammbuch (Vauban [1686, S. 14], Vilquin 1975, S. 256). Man kann diese Sätze des französischen Militärfachmanns, niedergeschrieben kurz vor Beginn der großen Eroberungsfeldzüge Ludwigs, durchaus als Mahnung an seinen Herrn lesen, die eigenen Staatskräfte realistisch einzuschätzen und nicht durch Kriege zu überfordern.

Es ist anzunehmen, dass sowohl Vauban (indirekt) als auch Leibniz (als Mitglied der Royal Society) wenigstens eine ungefähre Kenntnis davon besaßen, dass es in England mit John Graunt (1629-1674, Mitglied der Royal Society seit 1662) und Sir William Petty (1623-1687, Mitglied der Royal Society seit 1660) angesehene Gelehrte gab, die schon in den 1660er- und 1670er-Jahren Berechnungen und ta- 
bellarische Darstellungen zur demografischen und ökonomischen Stärke Englands nicht nur projektiert, sondern tatsächlich auch in Angriff genommen hatten. Schon in John Graunts Natural and Political Observations upon the Bills of Mortality (1662) findet sich der Grundgedanke, dass man die demografischen Basisdaten aus den Sterberegistern der Londoner City (Bills of Mortality), „,reduced into Tables“, dazu nutzen könne, ,so as to have a view of the whole together, in order to the more ready comparing of one Year, Season, Parish, or other Division of the City, with another" (Graunt 1662, S. 2). Graunt und sein Freund Petty gingen allerdings über die bloße Aggregierung und raumzeitliche Vergleichung des rohen Datenmaterials hinaus. Vielmehr ersuchten sie, aus ihren Zahlenreihen durch Rechenoperationen neue Erkenntnisse über Relationen zu gewinnen. Im Falle Graunts waren dies Relationen zwischen naturalen (etwa jahreszeitlichen) und sozialen (etwa gruppenspezifischen) Gegebenheiten einerseits, demografischen Effekten andererseits (z.B. Raten bestimmter Todesarten). Graunt und Petty waren damit die ersten wirklichen Statistiker im modernen Sinne (Behrisch 2016b, S. 243). Während Graunts Blick eher auf die Bevölkerungsentwicklung innerhalb Englands fixiert blieb, interessierte sich Petty hauptsächlich für die Wachstumsperspektiven Englands im Vergleich zu rivalisierenden Staaten, allen voran Holland und Frankreich. In diesem Geiste versuchte er 1670 erstmals eine Gesamtberechnung des englischen Nationaleinkommens, und zwei Jahre später verglich er dieses sowie die Bevölkerungsdichte mit den ihm verfügbaren entsprechenden Daten für die Nachbarstaaten, um zur Prognose zu gelangen, dass der englische Nationalreichtum in Zukunft denjenigen Hollands und Frankreichs übertreffen könne (Slack 2015, S. 120-123). Zwar publizierte Petty keine dieser Schriften zu Lebzeiten, doch brach die Debatte über Englands relativen Wohlstand und seine Wachstumschancen seitdem nicht mehr ab.

Der bedeutendste politische Arithmetiker der zweiten Generation, Gregory King (1648-1712), korrigierte Pettys Berechnungen zum Teil nach unten und warnte im Kontext der nach 1688 einsetzenden Kontinentalkriege vor der ,,vanity of overvaluing our own strength“ (zit. nach Slack 2004, S. 607). Kings Natural and Political Observations and Conclusions upon the State and Condition of England (1696) blieben ebenfalls zu Lebzeiten unpubliziert, doch wurde er wegen seines Zahlenwissens von zeitgenössischen Politikern häufig konsultiert, sodass seine Warnung vor leichtfertiger Fortsetzung der Kontinentalkriege nicht ungehört verhallt sein dürfte (Innes 2009, S. 121). Der letzte Paragraf von Kings Werk (\$ 13) ist unter dem Aspekt des zweckorientierten Staatskräftevergleichs besonders interessant. Er enthält für die drei Vergleichsländer England, Frankreich und Holland, bezogen auf die Jahre 1688 und 1695, Zahlenangaben in tabellarischer Form zum Einkommen pro Kopf, zur Steuerleistung pro Kopf und zu den Konsumausgaben pro Kopf sowie zu einem jährlichen Gesamtwachstum oder Gesamtrückgang. Das Resultat fasste King anschließend nochmals im Klartext zusammen. Für England und Frankreich ergab sich demnach zwischen 1688 und 1695, also seit Kriegsbeginn, ein Rückgang des jährlichen Einkommens, der für England nur geringfügig schwächer ausfiel als für Frankreich, während für den Handelsrivalen Holland ein Wachstum herauskam (King [1696]; Laslett 1973, S. 69). Direkte politische Schlussfolgerungen bot King an dieser Stelle nicht an, doch in einem Brief des gleichen Jahres an einen Mr. Stepney, den Gesandten des Königs an die Fürsten des Rheinlands, sprach er den 
Zweck seiner Berechnungen klar aus. Er habe „,den Effekt“ zeigen wollen, ,welchen dieser große und teure Krieg gegen Frankreich auf die Macht und den Wohlstand Englands haben könne, um so unsere Fähigkeit, diesen fortzusetzen und aufrechtzuerhalten, beurteilen zu können“" (King [1696], Laslett 1973, S. 171). Es ging ihm also um die Einschätzung des weiteren Durchhaltevermögens Englands im Krieg gegen Frankreich.

An modernen Maßstäben gemessen und für heutige wirtschaftshistorische Zwecke mögen die von King 1696 angestellten volkswirtschaftlichen Berechnungen unzureichend sein (vgl. Slack 2004, S. 626-628). Zeitgenössisch aber eilten sie der Literatur zum Staatskräftevergleich im 18. Jahrhundert weit voraus. Es ist daher wohl kein Zufall, dass Kings nur als Manuskript überlieferte Schrift im Jahr 1802 erstmals publiziert wurde, also erneut im Kontext eines drohenden Kriegs mit Frankreich. Der Herausgeber war George Chalmers, der schon 1782 mit einer Schrift über die Comparative Strength of Great Britain hervorgetreten war, in deren Neuauflagen (1802, 1804) er nunmehr Kings Schrift integrierte (Chalmers 1804).

\subsection{Beschreiben - Zählen - Vergleichen: Metamorphosen der „Statistik“ im 18. Jahrhundert}

Als das britische militärische Engagement auf dem europäischen Kontinent nach dem Frieden von Utrecht (1713) abflaute, entpolitisierte sich für einige Jahrzehnte auch die britische ,politische Arithmetik“. Das Methodenwissen ging zwar nicht verloren, aber die Anwendungsfelder verlagerten sich von der vergleichenden Staatskräftemessung auf andere Gebiete, darunter das Armenwesen, die Medizinalstatistik oder Untersuchungen zur Mortalitätswahrscheinlichkeit, die für die entstehende Lebensversicherungsbranche relevant waren (Innes 2009, S. 127-131; Rusnok 2002). Spätestens mit dem Siebenjährigen Krieg und der nach 1763 anhaltenden weltpolitischen Herausforderung durch Frankreich kehrte aber in Großbritannien das Interesse an einer Arithmetik politischer Macht wieder zurück, um bis zum Sieg über Napoleon nicht mehr zu erlahmen. Das erwähnte, mehrfach aufgelegte Werk von Chalmers zur Comparative Strength of Great Britain $(1782,1802,1804)$ war dafür ein Beispiel. Ebenso bewirkten der Siebenjährige Krieg und seine desaströsen Folgen für die Staatshaushalte auch in Frankreich und den deutschen Staaten einen Schub in Richtung einer effizienteren, statistisch gestützten Ressourcenerfassung und -mobilisierung (Behrisch 2016b, S. 246). Ziele waren die Bevölkerungsvermehrung, der innere Landesausbau, die Beförderung des Wohls der Untertanen (und damit auch des Fiskus) und schließlich nicht zuletzt die Steigerung der militärischen Stärke.

Vieles von dem, was deutsche Staatsdiener oder französische Intendanten des späteren 18. Jahrhunderts auf administrativer Ebene an Zählungen, Berechnungen und Schätzungen anstellten, blieb unterhalb der Schwelle öffentlicher Wahrnehmung. Die konkreten Zähl- und Rechenmethoden waren noch nicht routinisiert und interessierten nur einen kleinen Kreis von Beamten und Spezialisten. Die für Regierungsakteure und ein breiteres Publikum im In- und Ausland interessanteren aggregierten Ergebnisse wiederum unterlagen, soweit es um die Staatsfinanzen und den Militärapparat ging, im Prinzip der Geheimhaltung, zumal in Zeiten des Kriegs oder eines drohenden Kriegs - im späten 18. Jahrhundert also eigentlich immer. Dennoch gab 
es Mittel und Wege, an Kenntnisse über die relative Stärke von Rivalen oder Bündnispartnern zu gelangen. So nutzte Friedrich der Große nach dem Siebenjährigen Krieg Informationskanäle zum Amsterdamer Anleihenmarkt, um etwas über das credit rating potenzieller Gegner zu erfahren (Scott 2009, S. 44). Einer Sensation kam es gleich, als der französische Finanzminister Jacques Necker im Februar 1781, während des amerikanischen Unabhängigkeitskriegs, erstmals den französischen Staatshaushalt in seinem Compte rendu au Roi veröffentlichte. Als vertrauensbildende Maßnahme für mögliche Kreditgeber gedacht, löste die in 100.000 Exemplaren verkaufte Publikation in adligen Hofkreisen und bei den Kriegs- und Außenministern einen Skandal aus und kostete Necker letztlich sein Amt. Im Ausland hingegen erntete Necker auch Zustimmung. Der preußische Staatsminister Ewald Friedrich von Hertzberg ging in seinen publizierten jährlichen Reden vor der Berliner Akademie der Wissenschaften ab 1783 dazu über, im Anhang Zahlen zu den preußischen Staatsausgaben beizufügen und nannte 1785 explizit Neckers vertrauensbildenden Schritt als Vorbild (Klueting 1986, S. 262 f.).

Hertzberg war selbst an statistischen Fragen interessiert, allerdings an den konkreten Methoden nur insoweit, wie deren Ergebnisse dafür sorgten, dass der preußische Staat in der europäischen Rangfolge möglichst weit oben erschien. Seine Akademiereden dienten dem „Nachweis der Dauerhaftigkeit der Machtstellung Preußens“ (Klueting 1986, S. 247). Fiskal-militärische Stärke war für Hertzberg indes nicht das einzige Kriterium. Ihm ging es auch um den relativen Stand der Kultur. Er akzeptierte den von einigen akademischen Statistikern seit Mitte des 18. Jahrhunderts ins Spiel gebrachten Gedanken, dass die Bevölkerungsdichte ein Hauptkriterium zur Messung des allgemeinen Kulturzustands in einem Staate sei oder in den Worten von August Friedrich Wilhelm Crome (1753-1833), der zu den Verfechtern dieser Idee zählte: „daß die Cultur einer Nation, mit ihrer Volkszahl auf einen bestimmten Raum, ununterbrochen steigt und fällt" (Crome 1785, zit. nach Nipperdey 2015, S. 51). Da nun aber Brandenburg-Preußen nach diesem Kriterium in der Rangordnung der Staaten nicht sonderlich gut abschnitt, wandten Hertzberg und andere preußische Autoren ,alle möglichen Tricks an, um den Wert besser aussehen zu lassen“, etwa indem sie verlangten, dass (schwer messbare) naturräumliche Zusatzkriterien, wie die relative Fruchtbarkeit des Landes, in die Bewertung einzubeziehen seien; Hertzberg beteiligte sich also am „Spiel des Zahlenwettbewerbs“ (Nipperdey 2015, S. 55 u. 58). Zugleich versuchte er aber, bestimmte eingeführte Kriterien zu relativieren - ein frühes Beispiel für das Reagieren auf ein Staatenranking durch einen der verglichenen Akteure, und zugleich ein frühes Beispiel für die Ausweitung des Sets der Indikatoren von absoluter (demografischer, fiskaler, militärischer) Macht zu einer kulturell und ökonomisch verstandenen relativen Fortschrittlichkeit.

Publizierte Staatskräftevergleiche waren gegen Ende des 18. Jahrhunderts eine nicht mehr nur akademische Angelegenheit. Sie wurden sogar in andere europäische Sprachen übersetzt. So erschien beispielsweise 1786 eine deutsche Übersetzung von Chalmers' erwähntem Werk unter dem Titel Schätzung der verhältnismäßigen Stärke von Großbritannien (Chalmers 1786). Ein Jahr später publizierte ein deutscher Geograf und Zoologe, Eberhard August Wilhelm Zimmermann (1743-1815), eigens für ein britisches Publikum eine nur in englischer Sprache existierende Kompilation aus deutschsprachigen statistischen Werken unter dem Titel Political Survey 
of the Present State of Europe, in Sixteen Tables (Zimmermann 1787). Dies geschah mit dem erklärten Ziel, die britische Leserschaft mit den Resultaten einer seit etwa 40 Jahren in Deutschland florierenden, in England hingegen bisher vernachlässigten Wissenschaft, ,distinguished by the new-coined name of Statistics“, vertraut zu machen (Zimmermann 1787, Preface, S. ii). Durch Zimmermann und andere Vermittler gelangte der deutsche Begriff "Statistik“ als Neologismus nach England und zwar in der damaligen deutschen Bedeutung des Worts (Cullen 1975, S. 10).

Seit der Mitte des 18. Jahrhunderts wurde an immer mehr deutschen Universitäten „Statistik“ gelehrt, worunter keineswegs immer, nicht einmal in erster Linie zahlengestützte, tabellarisch präsentierte Berechnungen, sondern überwiegend beschreibende Darstellungen im Fließtext mit nur gelegentlich eingestreuten einfachen Tabellen oder Zahlenangaben verstanden wurden. Objekt der Darstellungen war stets ein konkreter „Staat“ (daher der Name „Statistik“), also der Zustand (lat. status) eines Herrschaftsverbands in einer Vielzahl von Dimensionen, die nur teilweise zähl- und messbar waren (Topografie, Flora, Fauna, Bodenprodukte, Gewerbe, politische Verfassung, Sitten, religiöse Verhältnisse, dann auch Einwohnerzahl, Flächengröße, Staatseinkünfte und Militär). „Statistik“ in diesem Sinne konnte sich nur auf einen Staat oder vergleichend auf mehrere europäische, gelegentlich auch außereuropäische Staaten beziehen. Ein führender älterer Vertreter einer derart weit verstandenen, fast ausschließlich verbal beschreibenden Statistik war der Göttinger Jurist und Historiker Gottfried Achenwall (1719-1772), den Zimmermann in seiner englischsprachigen Kompilation als einen seiner Gewährsleute zitierte (Achenwall 1749). Am anderen Ende des Spektrums, ebenfalls von Zimmermann als Autoritäten angeführt, standen jüngere Autoren wie der Braunschweiger Historiker Julius August Remer (1738-1803) oder der Gießener Kameralwissenschaftler August Friedrich Wilhelm Crome, die sich in ihren Werken der 1780er-Jahre weitgehend oder fast ausschließlich auf tabellarisch dargebotene Zahlenreihen oder, im Falle Cromes, grafische Darstellungen von Größenverhältnissen (Fläche, Einwohnerzahl, Bevölkerungsdichte) zu konzentrieren begannen (Remer 1786; Crome 1785).

Cromes Werk von 1785, berühmt geworden durch seine „Größen-Karte von Europa“ (dazu Nikolow 2001), entstand in einer Phase zwischen den großen Kriegen, als es ihm für einen Moment so schien, als seien Staatenvergrößerungen durch kostspielige Eroberungskriege nicht mehr möglich, weshalb jeder Fürst bestrebt sein müsse, die "Stärke und den Flor“ des eigenen Landes bestmöglich zu heben (Klueting 1986, S. 80-83). Die Bevölkerungsdichte erklärte Crome, wie erwähnt, zum ersten Maßstab des Fortschritts; seine Zahlen und grafischen Darstellungen sollten den europäischen Staatenvergleich unter diesem Aspekt auf einen Blick ermöglichen. Tatsächlich war Cromes Karte als Experiment zwar faszinierend, doch letztlich weitaus weniger leicht lesbar als die schlichteren Tabellen mit einfachen Zahlenangaben zu Flächengröße, Bevölkerungszahl und-dichte, Staatsfinanzen sowie Heeres- und Flottenstärke, wie sie seit den 1780er-Jahren vermehrt in den gedruckten Werken der Tabellenstatistik auftauchten.

Die für das britische Publikum kompilierte Political Survey of Europe von Zimmermann ist ein gutes Beispiel für die Reduktion auf wenige mess- und zählbare Größen, der für die deutschen Tabellenstatistiker des späten 18. und beginnenden 19. Jahrhunderts typisch war. Von den verbal-beschreibenden Staatenkundlern in der 
Göttinger Tradition Achenwalls wurden sie dafür im sogenannten Statistikerstreit (ab 1805) gescholten und als ,Tabellenknechte“ lächerlich gemacht (Schlözer 1808, zit. nach Twellmann 2015, S. 149). Die Vielzahl derartig vereinfachender Übersichten lässt aber auf ein gewisses Publikumsinteresse, ähnlich dem Interesse an heutigen Staatenrankings, schließen. So gestaltete Zimmermann die Tabellen in seinem ersten, den europäischen Staaten insgesamt gewidmetem Kapitel jeweils auf einer Seite oder Doppelseite und damit so knapp, dass sie für jeden Indikator (,Area“, „Population“, „Number of Inhabitants on each square mile“, „Public Revenue“, „Land Forces“, „Naval Forces“) auf einen Blick den Spitzenreiter und seine nächsten Rivalen erkennen ließen, auch wenn er die Tabellen noch nicht exakt als Rangordnung von Platz 1 absteigend bis x präsentierte. Bei den kriegsrelevanten Faktoren der Staatseinkünfte, Land- und Seestreitkräfte stach die Absicht, eine Rangfolge sichtbar zu machen, besonders hervor (Zimmermann 1787, S. 6-14). Allerdings handelte es sich hier, wie bei fast allen damaligen Tabellenstatistiken, um eine Momentaufnahme, im Fall Zimmermanns eine Erfassung des Zustands um die Mitte der 1780erJahre. Wegen ihres nichtseriellen Charakters erlaubten die Tabellenstatistiken je für sich noch keine temporalisierten Vergleiche.

Rangordnungen von Staaten etablierten die akademischen Statistiker, ob verbalbeschreibend oder numerisch-tabellarisch verfahrend, noch in einem weiteren Sinne: hinsichtlich der Zugehörigkeit zu einer Macht- oder Rangklasse. Julius August Remer beispielsweise unterschied in seinem Lehrbuch der Staatskunde (1786) drei Klassen von Staaten nach dem Grad ihrer Macht, die ,,herrschenden Staaten“ (England, Frankreich, Österreich, Preußen, Russland), die „mittlern Staaten“ (Türkei, Spanien, Niederlande, Dänemark, Schweden, Sardinien) und die ,kleinern Staaten“ (Portugal, Sizilien, Schweiz, Polen, Pfalz-Bayern, Sachsen, Venedig). „Alle übrigen“, so Remer, „kommen gar nicht in Erwegung, wenn die Rede von dem Staatsverhältnisse von Europa ist" (Remer 1786, zit. nach Klueting 1986, S. 60). Extremer noch auf die Kriegsfähigkeit bezogen war die - während des Siebenjährigen Kriegs publizierte - Klassifizierung in Jakob Friedrich Bielfelds Institutions politiques (1760). Bei ihm bestand die erste Klasse nur aus England und Frankreich, weil nur diese beiden Staaten einen Krieg allein, ohne Bündnispartner, durchstehen könnten; die zweite Klasse bildeten Österreich, Russland, Preußen und Spanien, da sie auf Bündnisse angewiesen seien; die dritte Klasse (Portugal, Sardinien, Schweden, Dänemark, Neapel, Niederlande) könne allenfalls mit Hilfstruppen in einen Krieg eingreifen; die Staaten der vierten Klasse schließlich (alle übrigen) könnten militärisch nichts ausrichten, sondern müssten sich durch Mäßigung und Klugheit zu behaupten versuchen (Klueting 1986, S. 128f.). Für den Duc de Choiseul, den ersten Minister Ludwigs XV., war im gleichen Jahr (1760) ein anderes Kriterienbündel entscheidend: Seemacht, Handel und Kolonialbesitz. Österreich, Russland und der König von Preußen seien demnach nur ,zweitrangige Mächte“, genau wie alle anderen, die einen Krieg nur führen könnten, wenn sie von den Kolonien besitzenden „kommerziellen Mächten“, also Frankreich, England, Spanien und Holland, Subsidien erhielten (Scott 2009, S. 47). Die Kriterien der Hierarchisierung variierten mithin je nach Autor und Schreibabsicht, in den meisten Fällen ergaben sich aber Dreier- oder Viererschemata der Rangklassen europäischer Staaten. 
Noch vor jeder Definition einer Rangordnung war zu klären, welche Einheiten überhaupt als „Staat“ zählen sollten, mithin Gegenstand einer „Statistik“ werden könnten. Der Göttinger Historiker Johann Christoph Gatterer fand darauf in seinem „Ideal einer allgemeinen Weltstatistik“ (1773) die Antwort, dass „,wilde Völker“ ebenso wie „Völker, welche andern Völkern unterthänig oder zinsbar sind“" nicht dazu gehörten. Gatterers Kriterium war also die uneingeschränkte Souveränität, auch wenn er das Wort nicht verwendete. Als „statistische Staaten“ in diesem Sinne ließ Gatterer in Europa nur „,19 bis 20“, in Asien nur ,4 bis 5“ (Persien, Mogulreich, Tibet, China, Japan) und in Afrika nur einen (Marokko) gelten; in Amerika schließlich gebe es „kein einziges statistisches Reich“ (Gatterer 1773, S. 16-19; vgl. Gierl 2015). Die Klassifizierung als Staat oder Nichtstaat und die Hierarchisierung der Staaten nach Rangklassen waren mithin untrennbar verbunden mit eben jenen „statistischen“ Staatskräftevergleichen, die im späten 18. Jahrhunderts zur gängigen Praxis wurden.

\subsection{Berechnungen für Krieg und Frieden: Koalitionen gegen Napoleon und Wiener Kongress}

Im Zeitalter der Kriege gegen das revolutionäre, dann napoleonische Frankreich setzten sich die Berechnungen der Kriegs- und Bündnisfähigkeit europäischer Mächte in den schon bekannten Mustern fort. Die erwähnte Erstpublikation der Pionierarbeit von Gregory King durch Chalmers (1802) gehört in diesen Zusammenhang. Auch die Übersetzungstätigkeit nahm an Fahrt auf. So übersetzte der schottische Ökonom William Playfair, der in seiner eigenen „Lineal Arithmetic“ (1798) und seinem „Statistical Breviary“ (1801) mit grafischen Veranschaulichungen statistischer Befunde, ähnlich wie Crome, zu experimentieren begann, im Jahr 1800 auch Jakob G. Boettichers Statistische Uebersichts-Tabellen aller Europäischen Staaten (1790) ins Englische (Cullen 1975, S. 11 f.; Innes 2009, S. 149 f.). Boettichers Tabellen zu den üblichen Größen (Volkszahl und-dichte, Fläche, Staatseinkünfte, Landund Seemacht) waren zwar nicht regelrecht als Rankings dargeboten, erlaubten es aber dem Leser, eigene Rangfolgen nach den verschiedenen Kriterien zu erstellen. Nach dem Frieden von Lunéville (1801) und dem in seinem Gefolge erlassenen Reichsdeputationshauptschluss (1803) kam im bald darauf aufgelösten Deutschen Reich eine weitere Funktion der kräftemessenden Statistik hinzu. Sie half nun bei der Zuschneidung von Territorien, die zwecks Bewahrung des künftigen Gleichgewichts und Friedens als Kompensationsmasse unter den verbliebenen größeren Mächten ohne Rücksicht auf historische Bindungen hin- und hergeschoben wurden (Klueting 1986, S. $291 \mathrm{ff}$. .).

Deutlich im Horizont kürzlich beendeter und neuerlich drohender Kriege verfasst und damit zeittypisch waren Johann Georg Heinrich Hassels Statistische Uebersichts-Tabellen der sämmtlichen Europäischen und einiger aussereuropäischen Staaten (1809). In seiner auf „November 1808“ datierten ,,Vorerinnerung“ beklagte Hassel, der kurz darauf zum Leiter des statistischen Bureaus des Königreichs Westphalen in Kassel ernannt wurde, dass die Statistik, wenn sie „den Ereignissen des Tages nacheilen“ wolle, ,in jedem Jahre eines neuen Kleides“ bedürfe. Deshalb habe er sich ,auf die äussern Umrisse beschränkt“ und nur das aufgenommen, 
was sich ,,auf den Flächeninhalt, die Volksmenge, Finanzen, Militär und Kolonien der Staaten“ beziehe. Auch in dieser Form dürften die Übersichten, so Hassel, „dem Publikum nicht unwillkommen seyn“ (Hassel 1809, o. S.). Die Anlage seines Tabellenwerks legte durchaus eine Lesart nahe, bei der ein potenzieller Krieg zwischen den großen Machtblöcken gleichsam antizipiert wurde. Schon das Inhaltsverzeichnis implizierte eine Rangordnung zwischen den namentlich aufgeführten großen Reichen (Kaisertum Frankreich, Französisches Reich, Kaisertum Russland, Kaisertum Österreich, Britisches Reich) und den namentlich nicht genannten „Staaten vom zweiten und dritten Range“, zu denen Hassel, wie dann erst im betreffenden Abschnitt klar wird, die Königreiche Preußen, Dänemark, Schweden, Sardinien, Sizilien sowie das Reich der Osmanen zählte. Den potenziellen Konflikt vorwegnehmend wirkt vor allem Hassels Zusammenfassung eines französischen Machtblocks, bestehend aus dem eigentlichen Kaisertum Frankreich und dem, was er das „Französische Reich“ nannte, worunter er die formal souveränen, de facto jedoch abhängigen Königreiche Italien, Neapel, Holland (mit Kolonien), Spanien (mit Kolonien), Portugal, die Rheinbundstaaten, den Kirchenstaat sowie Helvetien und das Wallis begriff. Vollends erkennbar wird Hassels Absicht im Schlusskapitel „G. Rekapitulazion des Europäischen Staatensystems“, in dem er aggregierte Gesamtzahlen für die Fläche, Volksmenge, Einwohnerdichte, Staatseinkünfte und Landmacht für ,,das französische Europa“ einerseits, „das übrige Europa“ andererseits einander gegenüberstellte, und zwar in der Weise, dass die Kräfteverhältnisse tatsächlich auf einen Blick zu sehen waren. Demnach übertraf das gesamte übrige Europa den französischen Machtblock in der Fläche - nicht verwunderlich - um etwa das Dreifache, bei der Landmacht immerhin noch um etwa 500.000 Männer (1,7 zu 1,1 Mio.), während es bei der Einwohnerzahl nur einen knappen Vorsprung besaß (94,9 zu 84,9 Mio.), auch bei den Einkünften kaum voraus war (685 zu 515 Mio. Gulden) und bei der Bevölkerungsdichte nur ein Drittel des französischen Werts erreichte (2399 zu 799 Einwohner pro Quadratmeile). Eine gesonderte Tabelle für die Seemacht wies aus, dass die Flotte des Britischen Reichs derjenigen aller übrigen europäischen Länder zusammen in sämtlichen Schiffsklassen, wenn auch nicht der Zahl der Matrosen, klar überlegen war (Hassel 1809, S. 38, 40). Eine politische Schlussfolgerung bot Hassel nicht an. Sein Zahlenwerk legte jedoch nahe, dass ein Landkrieg gegen das napoleonische Imperium wenn überhaupt, dann nur unter Aufbietung aller Kräfte außerhalb des französischen Machtblocks erfolgversprechend schien, während umgekehrt eine Seestrategie Napoleons zum Scheitern verurteilt war. Wären Hassels Tabellen im Februar 1809 in Wien zu Rate gezogen worden, als dort der Entschluss zum Krieg gegen Napoleon reifte, mit Unterstützungszusagen nur durch die Briten und Schweden, hätte die Kriegspartei am Wiener Hof, darunter der damalige österreichische Botschafter in Frankreich, Graf Metternich, vernünftigerweise innehalten müssen. Die Entscheidung fiel, wie man weiß, anders aus. Wenn es um die Auslösung eines Kriegs ging, zudem unter gefühltem Zeitdruck, besaßen statistische Staatskräftevergleiche trotz aller Publizität, die sie um diese Zeit bereits hatten, nur schwache Relevanz.

Als man wenige Jahre später in Wien über eine allgemeine europäische Friedensordnung beriet, erlangten statistische Befunde eine größere Bedeutung (vgl. Speich Chassé 2020). In der eigens für das Problem der Territorialausgleiche unter den Sie- 
germächten ins Leben gerufenen „Statistischen Kommission“ avancierte die reine Bevölkerungsgröße, die ,sogenannte Seelenzahl“, zum Hauptkriterium des Staatskräftevergleichs (Klüber 1815, S. 3 f.). Metternichs Instruktion vom 24. Dezember 1814 hatte noch vorgesehen, dass die „Bewertung“ (évaluation) der Bevölkerung „nicht lediglich nach dem bloßen Zahlenverhältnis“ (sous le simple rapport de quotité), sondern auch unter dem Aspekt „der Art oder Qualität“ (sous celui de l'espèce ou de qualité) erfolgen solle. Der Herausgeber der Kommissionsakten, Johann Ludwig Klüber, merkte hierzu jedoch an, dass dieser ,,zweite Punkt ... eigene Schwierigkeiten“ habe und „,von der Commission wenigstens vorerst noch ausgesetzt“ wurde (Klüber 1815, S. 8). Federführend bei der Zusammenstellung der Zahlen war der preußische Staatsrat Johann Gottfried Hoffmann, seit 1810 Leiter des dortigen Statistischen Bureaus. Für das Material zur Volksmenge in den zur Umverteilung anstehenden Gebieten, darunter das ehemalige Königreich Westphalen, griff man auch auf die Tabellen des königlich-westphälischen Statistikers Hassel zurück (Klueting 1986, S. 300f.; Klüber 1815, S. 12 ff.). Die zu Beginn des 19. Jahrhunderts in einer Reihe von Staaten institutionalisierte Statistik sollte mithin die Streitfragen des Kongresses lösen helfen.

Im Zentrum stand die ,polnisch-sächsische Frage“, also die neuerliche Aufteilung Polens und die Verkleinerung des Königreichs Sachsen, das bis zuletzt an Napoleons Seite geblieben war, zugunsten Preußens. „Es ist eine Art von Menschenhandel“, so notierte am 5. Februar 1815 der recht gut informierte sachsen-weimarische Verleger Carl Bertuch, der sich als Lobbyist der Vereinigung der deutschen Buchhändler beim Kongress aufhielt, in sein Tagebuch. Seine kritische Sicht hielt Bertuch allerdings nicht davon ab, im gleichen Eintrag den günstigen Verlauf der statistischen Beratungen für seinen kleinen Heimatstaat, das Großherzogtum Sachsen-Weimar, festzuhalten: „Unsere Angelegenheiten stehen gut. Oesterreich möchte uns nur 50.000 Menschen geben, wir betreiben aber 100.000“ (Bertuch [1814/15] 1916, S. $115 \mathrm{f}$.).

Bertuch war längst nicht der schärfste Kritiker des von den Diplomaten praktizierten Bevölkerungsaustauschs unter dem Gesichtspunkt der bloßen ,Seelenzahl“ - als gäbe es keine historischen, sprachlichen oder nationalen Bindungen, die Zugehörigkeit begründeten. Der rheinisch-katholische Romantiker und deutsche Patriot Joseph Görres, der sich wie viele seiner Zeitgenossen vom Wiener Kongress ein selbstbestimmtes und vereinigtes Deutschland erhofft hatte, klagte am 26. Januar $1815 \mathrm{im}$ Rheinischen Merkur über die „Folgen jenes herzlosen, statistischen Wesens, das in neurer Zeit so um sich gegriffen, und das jetzt fleißig in der niedergesetzten Commission getrieben wird, welche die Wiener im Scherze kurzweg die Seelenkommission nennen“ (zit. nach Twellmann 2015, S. 161). Auch in der Karikatur griff man das Thema auf, bezeichnenderweise unter Verwendung des Bildes vom ,Gleichgewicht der Mächte“, das durch eine Waage veranschaulicht wurde. Am 15. Mai 1815, während der „Hundert Tage“ Napoleons, erschien in der Bonaparte freundlich gesinnten Satirezeitschrift Le Nain Jaune eine Karikatur unter dem Titel „La Balance politique" (vgl. Gabriëls 2017). Sie verknüpft zwei Motive miteinander: auf der einen Seite die britischen Subsidienzahlungen an die Kontinentalmächte als Gegenleistung für deren Truppen, hier symbolisiert durch den rotgekleideten Herzog von Wellington, der schwere Goldbarren auf die linke Wagschale legt und dabei bemerkt, dass man nur 3 Schilling pro Kopf zahlen werde (,Nous ne les paierons que 3. Schellings 


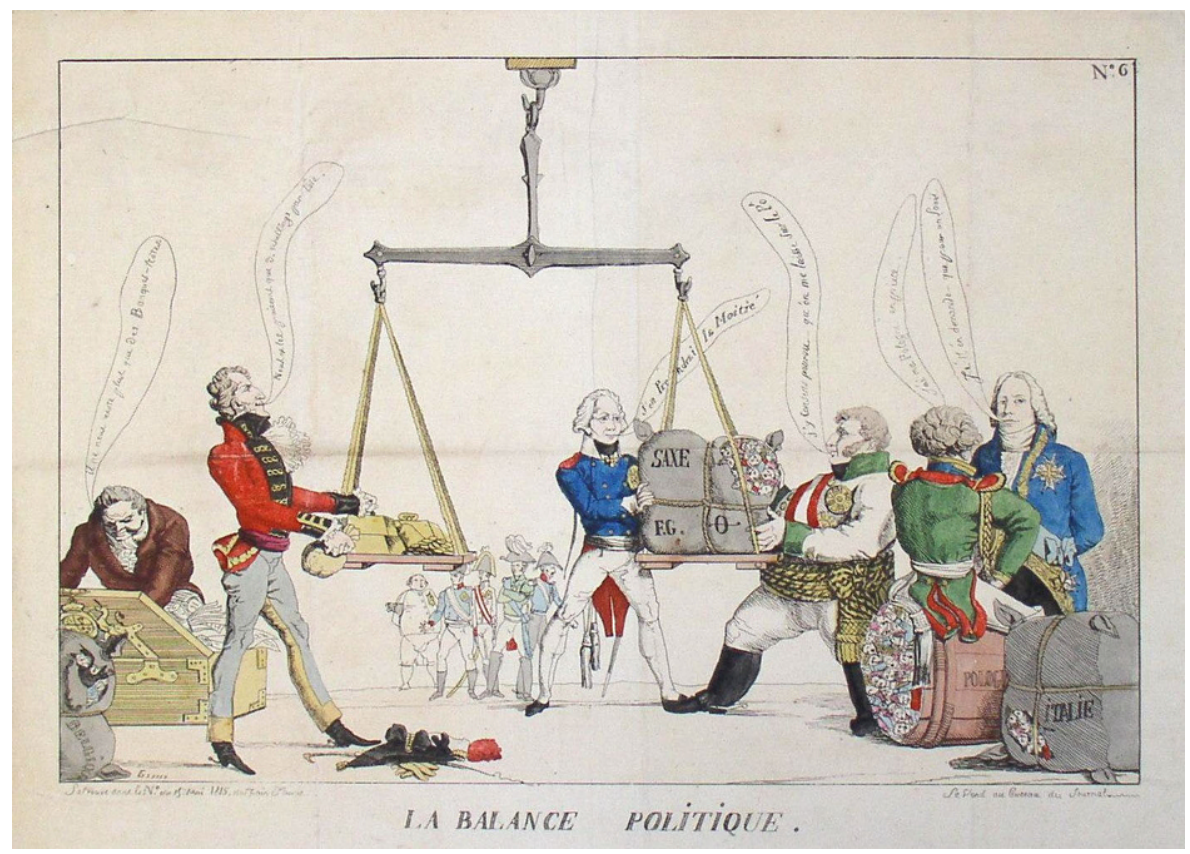

Abb. 1 La Balance politique, Le Nain Jaune, 15. Mai 1815, kolorierte Radierung, 27,1×40,3 cm, Bibliothèque Nationale de France, Dept. Estampes et photographies RESERVE QB-370 (73)-FT 4, https:// gallica.bnf.fr/ark:/12148/btv1b69546380/f1.item

par tête“); auf der anderen Seite das Geschacher der kontinentaleuropäischen Diplomaten um Territorien und ihre „Seelen“, veranschaulicht durch ein verschnürtes Paket „Sachsen“ (Saxe), aus dem an einer offenen Stelle Köpfe (Seelen) herausblicken. Das Paket wird vom König von Preußen (F. G., Frédéric Guillaume) und dem österreichischen Kaiser, der jedoch Züge Metternichs trägt, gemeinsam auf die rechte Waagschale gehievt. Dem Preußenkönig wird dabei mittels Sprechblase in den Mund gelegt, dass er die Hälfte nehme (,J'en Prendrai la Moitié“), worauf der Österreicher erwidert, er sei damit einverstanden, sofern man ihn an den Po, also nach Italien lasse (,j’y consens pourvu qu'on me laisse sur le Pô‘") (Abb. 1).

Italien wird durch ein weiteres verschnürtes Paket am unteren rechten Bildrand dargestellt, aus dem heraus wiederum Menschengesichter den Betrachter, geradezu Mitleid erheischend, anblicken. Ebenfalls am rechten Bildrand sitzt der russische Herrscher Alexander I. mit dem Rücken zum Betrachter auf einem Fass, das durch die Aufschrift als Polen („Pologne“) identifiziert wird. Auch das Fass ist gefüllt mit „Seelen“, die anscheinend unbemerkt vom Zaren aus der offenen Rückwand des Fasses zu entweichen suchen. Triumphierend verkündet der Zar, dass er nun „sein“ Polen am Stück ergattert habe (,,j’ai ma Pologne en pièce“), woraufhin sein Gegenüber, der französische Chefunterhändler Talleyrand, dies noch mit einem Fragezeichen versieht und als Gegenleistung vom Zaren die erneute Restauration des Bourbonenkönigs Ludwig XVIII. verlangt (,je m’en demande - que pour un Louis“). In dem der Karikatur beigefügten Kommentar kritisiert die bonapartistische, also an- 
tibourbonische Satirezeitschrift Le Nain Jaune die ,politische Börse“, die sich auf dem Wiener Kongress etabliert habe. Dort würden „Menschen und Staaten an den Meistbietenden verschachert“, und die Bevollmächtigten hätten sich ,,in Makler von Menschenfleisch transformiert“, die ,nach Belieben über das Leben, das Glück und die Freiheit von Millionen Menschen verfügen“ (zit. nach Gabriëls 2017, S. 145, meine Übers.). Rein statistische Staatskräftevergleiche im Stil des Ancien Régime gingen über die Lebensinteressen von Menschen und Nationen achtlos hinweg, so die Botschaft der Karikatur und des Begleittexts.

\section{Leistungsvergleiche von Staaten im 19. Jahrhundert: Erweiterung und Kritik}

Das Interesse an Staatenvergleichen ließ in der Friedensperiode nach 1815 keineswegs nach, verlagerte sich jedoch von der weiter fortlaufenden Berechnung der relativen Bevölkerungs-, Fiskal- und Militärstärke mehr und mehr auf Indikatoren, welche die Leistungsfähigkeit und den Zivilisationsgrad der Staaten anzeigten. Industrie, Handel, Bildung und Moral, ablesbar etwa an Kriminalitätsraten, gerieten ins Blickfeld der Statistiker und der um Prestige besorgten Regierungen. Nationale statistische Bureaus, die in den ersten Jahrzehnten des 19. Jahrhunderts überall in West- und Mitteleuropa errichtet wurden, sollten die Fortschritte des eigenen Landes diachron und synchron im Vergleich zu anderen Staaten dokumentieren. Seit 1851 bereicherten aufwendig inszenierte Weltausstellungen die inner- und zwischenstaatliche Vergleichspraxis um eine publikumswirksame visuelle Dimension. Bei aller Begeisterung für den Wettbewerb um Fortschrittlichkeit blieb auch Kritik nicht aus. Sie beschränkte sich nicht lediglich auf fehlerhafte Zählungen, schlecht gewählte Kriterien oder Darstellungsformen, sondern nahm bei einigen Autoren einen grundsätzlichen Charakter an. Trotz unterschiedlicher Ziele waren sich die Kritiker darin einig, dass sie die Reduktion der Vielfalt und je spezifischen Eigenart sozialen und nationalen Lebens auf statistische Größen zum Zweck der Vergleichbarmachung verurteilten. Gegen den Modus des von oben herab gleichmachenden, nur quantifizierenden Staatenvergleichs protestierten sie im Namen der Besonderheit oder sogar Einzigartigkeit von Individuen, Ständen, lokalen Gegebenheiten, sozialen Verhältnissen und Nationen. Diese Kritik setzte schon vor 1815 ein, weshalb dieser Abschnitt zum 19. Jahrhundert mit ihr beginnt, bevor anschließend die Praktiken der staatlichen Leistungsmessung und der Vergleichsinszenierung auf den Weltausstellungen vorgestellt werden.

\subsection{Plädoyers für das Singuläre: Kritische Stimmen gegen die Zahlenvergleiche}

Der schon erwähnte Streit, der in Deutschland um 1805 zwischen den Göttinger Statistikern der verbal beschreibenden Schule um August Ludwig Schlözer und den von diesen als „Tabellenknechte“ (Schlözer 1808) beschimpften Gegnern ausbrach, war mehr als nur ein akademischer Methodenstreit. Der Streit hatte eine tiefergehende, politische Dimension. Hinter der Kritik an den Tabellenstatistiken verbarg sich der Protest gegen die Konzeption eines Staats, der alles Individuelle, 
Zufällige, lokal Eigentümliche und historisch Gewachsene in numerisch erfassbaren Kategorien aufzulösen schien, um es leichter beherrschbar zu machen. Ob dieser angeblich alles nivellierende Staat in bürokratisch-absolutistischer Gestalt (wie in Preußen) oder revolutionär-zentralisierend (wie in Frankreich seit 1789) auftrat, war für die frühen Kritiker sekundär. Es waren wohl nicht zufällig Gelehrte und Beamte in ehemals kurhannoverschen Diensten, die nach dreimaliger Überwältigung durch einen fremden Machtstaat, zunächst Frankreich 1805, dann Preußen 1806, dann das artifizielle Königreich Westphalen unter Napoleons Bruder Jérôme ab 1807, besonders sensibel auf die gleichmachenden Effekte einer quantifizierenden und tabellarisch geordneten Statistik reagierten. Der hannoversche Regierungsrat und Revolutionsgegner August Wilhelm Rehberg (1758-1836) hatte sich schon 1793, inspiriert durch Edmund Burkes Diatriben gegen die ,,sophisters, oeconomists, and calculators“ des neuen Zeitalters (Burke [1790] 1989, S. 127), skeptisch über eine nur zählende Statistik geäußert, die den „Geist“ und „Charakter der Menschen“ nur unvollkommen erfasse. Reisebeschreibungen seien für diesen Zweck besser geeignet. Von den Preußen suspendiert und im Königreich Westphalen auf eine subalterne Beamtenposition abgeschoben, verschärfte Rehberg seine Kritik gegen eine auf Tabellenstatistiken gestützte Staatsverwaltung und wurde darin sekundiert durch den Göttinger Geschichtsprofessor Arnold Hermann Ludwig Heeren, der sich 1807 in einer zustimmenden Rezension zu Rehberg über die ,ewigen Wiederholungen von Flächeninhalt und Volksmenge, von Einkünften und Truppenzahl“ der ,Tabellenmacher“ beschwerte. „Für Nationalgeist, Freyheitsliebe, das Genie und den Charakter großer oder kleiner Männer an der Spitze, gibt es keine Columnen“; so Heeren in einer anderen Rezension 1806. Der aus dem hannoverschen Staatsdienst entlassene Burkeaner Ernst Brandes äußerte sich ähnlich wütend über die ,,aus dem Schlamme des Maschinenwesens“ hervorgegangenen „Zahlenstatistiker und Staatstabellen“ (vgl. Twellmann 2015, S. 150-159, dort auch die Zitate).

„Geist“, „Leben“, „Charakter“, „Genie“ einzelner Menschen und Nationen: Das waren die Stichworte einer Kritik, die sich gegen die Zurichtung vielgestaltiger Wirklichkeit auf wenige Kategorien zum Zweck des Zählens, Berechnens und rationellen Regierens wandte. Diese Stichworte wurden in den folgenden Jahrzehnten vielfach variiert, etwa durch die schon zitierten Angriffe auf die „Seelenkommission“ (Görres) während des Wiener Kongresses 1815. Weitere Gegensatzbegriffe kamen hinzu, so wenn der im Alter katholisch-konservativ gewordene Friedrich Schlegel in seiner Artikelserie Signatur des Zeitalters (1820-23) das ,lebendig Gegliederte“ als „organisches Wirken und Verfahren“ dem ,,atomistischen Zustande“ gegenüberstellte, der „das Wesen alles revolutionären Beginnens“ sei. Schlegel wandte sich gegen die Idee einer alles regierenden „Gesetzesmaschine ..., die umso vollkommener sei, je mehr alles Leben numerisch verzeichnet, alle Menschenkraft tabellarisch geordnet und eingetragen, nach dem gleichen Takt kunstreicher Zentralverwaltung sich ununterbrochen fortbewege." (Schlegel [1820-23] 1966, S. 539 u. S. 546). Trotz unterschiedlicher politischer Programmatik waren sich Autoren wie Rehberg, Görres oder Schlegel darin einig, dass sie eine Statistik ablehnten, die wesentlich Ungleiches gleichmachte, also „organisch“ und historisch Gewachsenes in seine einzelnen Bestandteile (,Atome“) zerlegte, um diese dann zählen und vergleichen zu können. Die Kritiker waren dabei nicht gegen Vergleiche (zwischen Staaten oder anderen Einhei- 
ten) als solche, aber sie verlangten Vergleiche, die primär auf Singularität abstellten und daher eher auf beschreibende statt auf numerische Darstellungen zurückgreifen sollten. Mit Recht sieht Alain Desrosières in der Forderung, Dinge in ihrer Ganzheit (und damit Eigenart) zu beschreiben, einen seit Beginn des 19. Jahrhunderts immer neu aktualisierbaren Modus der Statistikkritik: „In every case, a form of singularity (historical, national, individual) is appealed to, referring back to ways of describing things - in other words, of constructing totalities, different from those of statistics“" (Desrosières 1998, S. 22).

Waren es in der Restaurationsära der 1810er- und 1820er-Jahre vorwiegend Antirevolutionäre, Altkonservative, Nationalromantiker oder Verfechter christlicher Ständestaatsideen, die sich derart äußerten, gab es seit dem Vormärz auch Stimmen auf der radikaldemokratischen und sozialreformerischen Seite des politischen Spektrums, die in eine ähnliche Richtung zielten. So forderte 1831 der Radikaldemokrat Wilhelm Schulz (1797-1860) unter Berufung auf Schlözer, dass die Statistik „,den Zustand der Staaten ... in seiner Totalität zur Kunde zu bringen“ habe und sich daher nicht wie in den Zeiten der ,früheren Kabinettspolitik“ lediglich auf die ,,materiellen Staatskräfte" beschränken dürfe. Die offiziellen statistischen Jahrbücher müssten durch eine kritische ,periodisch-statistische Tagesliteratur“ ergänzt und korrigiert werden, in der ,die Thatsachen des öffentlichen Lebens in ihrem Zusammenhange geschildert" würden (Schulz 1831, S. 1, 4; vgl. Eiden-Offe 2015, S. 173-178). In seinem 1843 im Schweizer Exil verfassten Artikel „Statistik“ für das Staatslexikon von Rotteck und Welcker trat Schulz erneut für eine Statistik ein, die sich nicht auf ein „,bloses Aneinanderreihen von Ziffern und Zahlen“ beschränken solle, sondern sich vielmehr ,die Schilderung des Geistes einer Gegenwart“"vornehmen solle. Neben der tabellarischen Statistik und der „Linearmethode“, das heißt grafischen Visualisierungen, sollte für ihn auch eine ,ethnographische“ Darstellungsform, „die einen Staat nach dem anderen als besonderes Ganzes behandelt“, ihren Platz in der Statistik behalten. Auch der Fokus der Statistik müsse sich verändern. Der ,offiziellen und offiziösen Statistik“ setzte Schulz eine „oppositionelle Statistik“ entgegen, welche ,die Interessen und die Lage der unteren Volksclassen“ besonders zu beachten habe. Wo die offizielle Statistik aus dem Wachstum des Nationaleinkommens auf die „Zunahme des Wohlstandes im Ganzen“ geschlossen habe, solle die oppositionelle Statistik den Blick auf dessen zunehmend ungleiche Verteilung lenken, indem sie ,die Größe des Arbeitsverdienstes, im Vergleiche mit den Preisen der nothwendigsten Lebensbedürfnisse; die gewöhnliche tägliche Arbeitszeit; so wie die Sicherheit und Dauer des Arbeitseinkommens, oder vielmehr die Unsicherheit und die Schwankungen desselben“ ins Auge fasse (Schulz 1843, S. 829, 832f.).

Während Autoren wie Schulz in den 1840er-Jahren noch bemüht waren, den Begriff ,Statistik“ im Anschluss an Schlözer so weit zu dehnen, dass er quantifizierende sowohl als verbal-beschreibende (ethnografische, sozialkritische) Verfahren einschloss, wurde dies im postrevolutionären Zeitalter des Realismus und Positivismus ab 1850 schwieriger. Auf der einen Seite begann sich die Statistik als ,,selbständige Wissenschaft“" zu definieren, die - in den Worten des Nationalökonomen Carl Knies - nur das ,exacte Maß der Ziffer“ gelten lassen, ,überall objective Beweise geben“ und „niemals eine blos subjective Überzeugung hervorrufen“ wollte (Knies 1850, S. 84 u. 148; zit. nach Scheu 2015, S. 199). Auf der anderen Seite etablierte 
sich um die gleiche Zeit eine an Einzelfällen interessierte, je nach politischer Couleur der Autorinnen und Autoren volkskundlich (Wilhelm Heinrich Riehl) oder sozialreformerisch inspirierte Sozialreportage (Fréderic Le Play, Henry Mayhew, George Eliot) als eigenes literarisches Genre. Die Mehrzahl dieser Studien war auf Zustände im eigenen Land gerichtet, sie konnten aber auch ländervergleichend angelegt sein, so etwa im Fall von Le Plays Studie über die europäischen Arbeiter (1855). Doch statt auf Massenbeobachtung und Durchschnittswerte im Sinne der Moralstatistiken Adolphe Quételets zu zielen, setzte Le Play auf anschauliche Falldarstellungen, z. B. familienmonografische Budgetanalysen (Scheu 2015, S. 202-208). Methodisch und begrifflich trennten sich um 1850 die Wege zwischen einer streng quantifizierenden und berechnenden „Statistik“ im heutigen Sinne einerseits und einer qualitativen Sozialforschung andererseits, in der Zahlen und Berechnungen nur ergänzend eingesetzt wurden.

\subsection{Fortschritte zählbar machen: Ausweitung der nationalen und vergleichenden Statistik}

Mit dem Abflauen der Kriegsgefahr nach dem Wiener Kongress richtete sich das Interesse der amtlichen und gelehrten Statistik stärker als in den Jahrzehnten zuvor auf den jeweils eigenen Staat. Der äußere Staatenvergleich unter dem Gesichtspunkt des fiskal-militärischen Kräftemessens trat für eine längere Periode hinter die innere Reorganisation der Staaten zurück. Insbesondere dort, wo, wie in Mitteleuropa, die Grenzen verschoben worden waren und bisher fremde Bevölkerungen in die Staatsverbände integriert werden mussten, wurden die im ersten Drittel des 19. Jahrhunderts fast überall entstehenden statistischen Bureaus selbst zum Instrument der inneren Staatsbildung. Es galt, die neu zugeschnittenen Territorien administrativ $\mathrm{zu}$ durchdringen, die Behördenapparate und-verfahren zu vereinheitlichen und die Loyalität der Bevölkerungen sicherzustellen. Antirevolutionäre Repression im Stil Metternichs genügte dafür auf Dauer nicht, eine vorwiegend nur fiskalische und militärische Erfassung der Untertanen wie im Ancien Régime schon gar nicht. Der Nachweis gewisser Leistungen des Staates für seine Bürger musste hinzukommen. Die Statistik hatte diesen Nachweis zu erbringen.

Als Minimalerfordernis gehörte zum Aufgabenportfolio des Staates die Garantie der äußeren und inneren Sicherheit, vor allem der Schutz des Privateigentums, darüber hinaus das glaubwürdige Bemühen, den Wohlstand des Landes mehren zu wollen: durch Förderung von Landwirtschaft, Handel, Gewerbe und Verkehr sowie eine funktionierende Justiz. In Kontinentaleuropa fühlten die Staaten, anders als in England, auch bereits in den frühen Jahrzehnten des 19. Jahrhunderts eine gewisse Verantwortung für eine rudimentäre Schulbildung der breiten Masse und die höheren Bildungsanstalten der Eliten. In den mittleren Jahrzehnten des 19. Jahrhunderts drängte sich dann überall die Bewältigung der Folgelasten von Industrialisierung und Urbanisierung (,,soziale Frage“, Hygiene und Infrastruktur in den großen Städten) in den Gesichtskreis der europäischen Regierenden und ihrer Bürokratien.

Die statistischen Bureaus fanden ihre Existenzberechtigung darin, dass sie die Leistungen des Staates in diesen Feldern sukzessive dokumentierten. Wichtiger als der (synchrone) Vergleich mit dem Ausland war dabei im gesamten 19. Jahrhundert, 
vor allem aber in den ersten Jahrzehnten nach 1815, der (diachrone) Vergleich zu früheren Zeiten im Inland. Der Fortschritt im eigenen Land sollte in eingängigen Zahlenreihen sichtbar werden, die sich als stetige und möglichst anhaltende Bewegung zum Besseren interpretieren ließen. Die Statistik fungierte hier nicht nur als beobachtende Instanz, sondern als treibender Faktor, indem sie innerbehördlich, z. B. zwischen verschiedenen regionalen Verwaltungseinheiten, Wettbewerbe um möglichst vorteilhafte Zahlen, die man ,nach oben melden“ konnte, in Gang setzte. In den bürokratisch-absolutistischen Staaten Mitteleuropas waren also die Beamtenapparate zugleich Datenproduzenten und das primäre Publikum. Voraussetzung für die Effekte der Leistungsdokumentation und Wettbewerbssteigerung war eine regelmäßige Publikation entsprechender statistischer Daten (Serialität). Sie wurde in den statistischen Staatsapparaten im Laufe des 19. Jahrhundert allmählich zur Norm. Ob (und ab wann) die Publikation in jährlichen Abständen für eine festgelegte Zahl von Indikatoren geschah (statistische Jahrbücher), ob (und bei welchen Sachverhalten) die Publikation in größeren Intervallen erfolgte, wie z. B. im Fall des seit 1801 alle 10 Jahre erhobenen britischen Zensus, hing von der Dringlichkeit der jeweiligen Thematik, der Komplexität des Aufwands bei der Datenerhebung und den verfügbaren administrativen Kapazitäten ab.

Ausmaß und Grad der öffentlichen Verfügbarkeit staatlich generierter statistischer Daten variierten in den ersten Jahrzehnten des 19. Jahrhunderts innerhalb Europas erheblich. In Großbritannien war die Publizität von Anfang an schon dadurch garantiert, dass das seinerseits unter öffentlicher Beobachtung stehende Parlament als (meist zurückhaltender) Geldgeber, gelegentlich auch direkter Auftraggeber sowie durchgehende Kontrollinstanz nicht zu umgehen war. Am anderen Ende des Spektrums stand ein spätabsolutistischer Staat wie das Kaisertum Österreich, in dem ein zentrales statistisches Bureau nicht nur erst relativ spät eingerichtet wurde (1829; zum Vergleich: Preußen 1805), sondern der zudem eine äußerst restriktive Publikationspolitik verfolgte: Die nach den 15 Provinzen des Kaiserreichs gegliederten Zusammenstellungen der Daten standen vollständig nur in einer sehr wenigen Empfängern vorbehaltenen „Hofausgabe“ zur Verfügung. Schon die Zentralbehörden bekamen nur eine um sensible Militär- und Finanzdaten bereinigte knappere Version zu sehen und die Unterbehörden und die Öffentlichkeit mussten sich mit äußerst summarischen Auskünften begnügen, mit dem Nebeneffekt, dass die Lieferanten der Rohdaten auf der unteren Verwaltungsebene nur geringe Motivation verspürten, die aufwendige - für sie unsichtbar bleibende - Datenerhebung zu leisten. Erst nachdem Karl von Czoernig im Jahr 1841 die Leitung der „k.k. Direction der administrativen Statistik“ übernommen hatte, gelang es diesem nach mehrjähriger Überzeugungsarbeit, dem Kaiser ab 1845 zumindest die Veröffentlichung der volkswirtschaftlich relevanten Teile der Statistik abzuringen (Göderle 2018, S. 186-193).

Der höhere Grad an öffentlicher Sichtbarkeit und parlamentarischer Kontrolle in Großbritannien war auf der anderen Seite erkauft mit einer erheblichen Unübersichtlichkeit bei der statistischen Datenerhebung. Von wenigen früh und dauerhaft etablierten Serien wie dem Zensus abgesehen (vgl. Glass 1973, S. 90-105), war in der gesamten viktorianischen Ära eine Vielzahl von Ministerien und durch Gesetzgebung geschaffener Boards damit befasst, für die je eigenen Bereiche nach eigenen Relevanzkriterien mehr oder weniger regelmäßig Statistiken zu erstellen, 
nicht zuletzt in der Absicht, vor dem stets knauserigen Parlament die Notwendigkeit fortlaufender, wenn möglich höherer Finanz- und Personalzuweisungen zu rechtfertigen. Ein zentrales statistisches Bureau, in dem alle Daten gesammelt und aufbereitet wurden, gab es nicht. Nach der Wahlrechtsreform von 1832 wuchs zwar das Bewusstsein, dass Reformen, wie sie nun verlangt wurden (Armenrecht, Fabrikgesetze, Gesundheitswesen), ohne statistische Informationen schwer zu planen waren, doch das 1832 eingerichtete Statistical Department at the Board of Trade, dazu gedacht, als eine Art Sammel- und Koordinierungsinstanz der an vielen Stellen stattfindenden Datenproduktion zu dienen, erlangte zu keiner Zeit die nötige Autorität, um die anderen Ministerien und Boards zur Preisgabe ihres Herrschaftswissens für den jeweiligen Bereich oder gar zur Anpassung an gemeinsame Standards und Kategorien zu bewegen (vgl. Cullen 1975, S. 14-25). Stattdessen wurde 1837 mit dem General Register Office eine weitere konkurrierende Behörde mit dem Auftrag statistischer Erhebungen in den Feldern des Personenstandswesens, der Gesundheit und des Sozialen eingerichtet, die ab 1840 auch den Zensus übernahm (Desrosières 1998, S. 167 ff.). Das öffentliche Interesse an Statistik wuchs derweil stark an, was sich 1834 in der Gründung der Statistical Society of London (später Royal Statistical Society) und zahlreichen provinziellen Statistical Societies zeigte, die sich als Lobbygruppen für bessere Statistiken verstanden (Cullen 1975, S. 77-130). Die Klagen über das Fehlen eines zentralen statistischen Bureaus nahmen in Großbritannien seit Mitte der 1830er-Jahre zu, wobei vor allem die in Belgien 1841 unter der Leitung von Adolphe Quételet eingerichtete Statistische Zentralkommission als vorbildlich genannt wurde.

Die konträren österreichischen und britischen Beispiele verdeutlichen, wie hoch die praktischen Hürden waren, die schon auf innerstaatlicher Ebene überwunden werden mussten, um einen dem Aufgabenzuwachs der Staaten sich anpassenden, nach konsistenten Kriterien operierenden und den Erwartungen des Publikums (sowie der Regierenden und Bürokratien selbst) gerecht werdenden statistischen Apparat aufzubauen. Im österreichischen Fall verhinderte eine misstrauische Staatsführung lange die nötige Publizität und untergrub damit die Motivation der Datenproduzenten an der Basis, im britischen Fall sorgte ein misstrauisch über seine Kompetenzen und das Geld der Steuerzahler wachendes Parlament dafür, dass keine zentrale staatliche Behörde entstand, bei der statistisches Wissen monopolisiert wurde. Wenn der Aufbau einer statistischen Infrastruktur bereits innerhalb der Einzelstaaten auf so große Hindernisse stieß, um wieviel schwieriger musste es sein, die nationalen Sonderwege der Herstellung statistischen Wissens wenigstens soweit aufeinander abzustimmen, dass zwischenstaatliche Vergleiche möglich wurden?

Die Geschichte der Internationalen Statistischen Kongresse, die vor allem auf Betreiben des Belgiers Adolphe Quételet ins Leben gerufen wurden (der erste Kongress fand 1853 in Brüssel statt) und sich die Realisierung einer vergleichend-europäischen Statistik auf die Fahnen schrieben, ist - so gesehen - eine Geschichte des Scheiterns. Die Kongresse in Brüssel (1853), Paris (1855), Wien (1857), London (1860), Berlin (1863), Florenz (1867), St. Petersburg (1872) und Budapest (1876) schärften zwar immerhin das Bewusstsein für die technischen, methodischen und sprachlichen Probleme, so etwa das Erfordernis einer internationalen statistischen Fachterminologie (Randeraad 2010, S. 52, 138). Auch sorgten sie für die transnationale Vernetzung 
unter den staatlichen und akademischen Statistikexperten und erhöhten, besonders im jeweiligen Gastgeberland, deren Prestige. Wo noch kein zentrales statistisches Bureau bestand, so besonders in den konsolidierten alten Nationalstaaten Großbritannien und Frankreich, dienten die Kongresse zudem als Resonanzraum, um entsprechenden Forderungen auf nationaler Ebene Gehör zu verschaffen (Randeraad 2010, S. 52). Doch allein schon das Format des Kongresses selbst erwies sich wegen der zu hohen Zahl der Partizipanten, der unregelmäßigen Tagungsrhythmen und des Fehlens eines dauerhaften administrativen Unterbaus als ungeeignet, das proklamierte Ziel der Vereinheitlichung auch nur exemplarisch einmal umzusetzen. Eine Lehre daraus war der in den 1880er-Jahren vollzogene Wechsel zu einer anderen Organisationsform, dem 1885 in London gegründeten International Statistical Institute. In diese Richtung wies bereits Quételets Vorschlag auf dem Londoner Kongress von 1860, dass ein kleines Komitee, bestehend aus den prominentesten staatlichen Statistikern aus den beteiligten Ländern, damit betraut werden solle, die wichtigsten Zahlenreihen der nationalen Statistiken an einem Punkte zusammenzuführen. Der Vorschlag wurde ähnlich wiederholt vom preußischen Chefstatistiker Ernst Engel in Berlin 1863, vom Italiener Cesare Correnti in Florenz 1867 und bei allen weiteren Treffen. Es blieb aber bei Resolutionen (Randeraad 2010, S. 6f., 92, 118, 132).

Obwohl also die Kongresse ihr eigentliches Ziel, die Vereinheitlichung der einzelstaatlichen Statistiken zwecks internationaler Vergleichbarmachung, verfehlten, trieben sie die Ausweitung der Statistik (nun stets im quantifizierenden Sinne verstanden) auf immer neue Felder entscheidend voran. Die Kongresse bildeten das Forum und Publikum zugleich, vor dem die jeweiligen nationalen Statistiker sich zu überbieten suchten. Die vorbereitenden Komitees der Gastgeber suchten gezielt nach Sachgebieten, in denen sie mit ihren praktisch-statistischen Erfahrungen vor den anderen Teilnehmern glänzen konnten. Und dies waren sehr oft neue Themen: In Paris 1855 war es die Erfassung der Hygieneprobleme in den großen Metropolen, in Wien 1857 die Zählung und Kartierung ethnisch gemischter Bevölkerungen, in London 1860 die Statistik in den Kolonien und im Bereich des öffentlichen Gesundheitswesens, in Berlin 1863 die Einbeziehung der Statistiken genossenschaftlicher Unterstützungskassen und Versicherungen in amtliche Zählungen, in Florenz schließlich 1867 die Ausdehnung der staatlichen Statistik auf den bisher wenig beachteten kulturellen Bereich, also Schulen, Archive, Bibliotheken und Museen (Randeraad 2010, S. 53 ff., 64-69, 91-95, 122 ff., 141 ff.). Die Fortschritte der Statistik auf diesen Gebieten in einem Staat erhöhten den Druck in den anderen Staaten, diese auch dort zum Gegenstand amtlicher Zählungen und Berechnungen zu machen. Für potenzielle künftige Staatenvergleiche vermehrte sich damit das verfügbare Zahlenmaterial. Auch war es bei diesen für die Statistik relativ neuen Feldern leichter, sich auf basale gemeinsame Standards zu verständigen, als in den Gebieten, die schon früh zum Gegenstand einzelstaatlicher Statistiken geworden waren (Bevölkerungszensus, Handel, Steuern). Über die Art und Weise etwa, wie ein Zensus anzulegen sei, insbesondere über die Kategorisierung der zu zählenden Gruppen, konnten sich die auf den Kongressen versammelten Statistiker zu keiner Zeit einigen, obwohl sie über den Sinn uniformer Kriterien völlig einer Meinung waren. Die Ausweitung einer (potenziell) staatenvergleichenden Statistik auf Leistung und Kultur seit der Mitte des 19. Jahrhunderts war mithin ein nicht unwichtiger Nebeneffekt der Kongresse. 
Mangels einer staatenübergreifend Daten sammelnden und verarbeitenden Organisation, eines centre of calculation also (Speich Chassé 2019), blieb es bis ins späte 19. Jahrhundert dabei, dass statistisch basierte Staatenvergleiche, ob sachlichneutral oder in bewertender Absicht (als Ranking), im Wesentlichen nur von Einzelautoren durchgeführt wurden. Eher konventionell und in der vorwiegend verbalen Darstellungsform noch nah an den deutschen ,statistischen“ Werken des 18. Jahrhunderts war ein Werk wie Joseph Constantin Bisingers Vergleichende Darstellung der Grundmacht oder der Staatskräfte aller europäischen Monarchien und Republiken (1823). Thematisch enthielt es neben den üblichen Ausführungen zu FlächengröBen, Bewohnern, Verteidigungskräften und Finanzen immerhin längere Abschnitte über „Industrielle Production“, „Commercielle Produktion“ sowie „Geistescultur“ (Schulen, Wissenschaften, Zeitschriften, Buchhandel). Es fügte sich damit ein in den Trend zur Ausweitung des Staatenvergleichs von der bloßen Macht auf Leistung und Kultur. Eingestreut finden sich hier auch für einzelne Parameter Versuche, zu einem Ranking zu gelangen, so eine tabellarische „Rangordnung der europäischen Staaten in Rücksicht ihrer Einkünfte" nach absoluten Zahlen und - interessanter eine „Berechnung des Beytrages eines jeden einzelnen Individuums in den europäischen Staaten zu den Einkünften derselben“. Nach dieser Berechnung rangierte der „Britte“ mit Abstand auf dem ersten Platz, danach folgten der „Niederländer“, „Franzose“, „Marinese“ (!), „Deutsche“, „Russe“ usw. und schließlich auf dem letzten Platz 23 - man glaubt es kaum - der „Schweizer“. Bisinger selbst scheint dieses Resultat unplausibel vorgekommen zu sein, denn er schreibt, dass diese Berechnung „nicht zuverlässig“ sei, ,weil sie nur auf dem Verhältnisse der Bevölkerung zu der Staatseinnahme beruhet" (Bisinger 1823, S. 402-406); im Fall der Schweiz kam der schlechte Platz in Bisingers Ranking dadurch zustande, dass, wie er bemerkt, die Schweiz als Gesamtstaat gar „,keine Staatseinkünfte“ hatte (S. 405), ihm aber offenbar Angaben zur Gesamteinnahme aller Kantone fehlten. Das Beispiel verweist auf die Grenzen, auf die in den ersten Jahrzehnten des 19. Jahrhunderts einzelne Autoren stießen, wenn sie sich trotz fehlender oder nur partiell publizierter oder aber inkommensurabler gesamtstaatlicher Statistiken um Staatenrankings bemühten.

Die allmähliche Verbesserung der (national-)staatlichen Statistiken um die Mitte des 19. Jahrhunderts änderte daran grundsätzlich nichts. Nur in Teilbereichen weiter als Bisinger gelangte etwa der Statistiker Friedrich Wilhelm von Reden (1802-1857). Er war zunächst im hannoverschen, dann preußischen Staatsdienst tätig gewesen, hatte 1846 den Verein für deutsche Statistik mitbegründet und musste nach seiner Mitgliedschaft in der gemäßigt-linken Fraktion Westendhall der Frankfurter Paulskirche und dem Scheitern der Revolution seine vergleichend-statistischen Interessen als freier Autor fortführen. Seine 1848 in die Revolution hinein publizierte, unvollendet gebliebene Vergleichende Kultur-Statistik ... der Gross-Staaten Europa's beschränkte sich, anders als der Titel versprach, auf die üblichen Themen der Flächenund Bevölkerungsverhältnisse, Bodenbeschaffenheit und Bodenerzeugnisse sowie zum Schluss einige wenige, nur unsystematisch mit Zahlen unterfütterte Bemerkungen zu Lebensweise, Gesundheitszustand, Beschäftigungsweise und Arbeitsverhältnissen (Reden 1848). Als Wiederaufnahme und Fortsetzung brachte Reden dann im Jahr 1854 unter dem Titel Deutschland und das uebrige Europa ein über 1000-seitiges vergleichend-statistisches „Handbuch“ heraus. Bodenbeschaffenheit, Fläche, 
Demografie, Staatshaushalt und Streitmacht bildeten auch hier die klassischen Themen; dazu kamen nun umfangreiche, teilweise auch staatenvergleichende Ausführungen und Tabellen zu Produktion, Erwerbs- und Verkehrsverhältnissen (Straßen, Eisenbahnen, Telegrafen) sowie Handel (Einfuhr/Ausfuhr, Zölle). Kulturelle und moralische Aspekte hingegen blieben weiterhin ausgespart. Fast resignativ klang das Vorwort des Werks, in dem Reden die Leser auf die ,gedrängteren Vergleichungen und Verhältniszahlen“ hinwies, die sie hinter den „Urziffern“ und „unendlichen Ziffernreihen" finden könnten, zu deren ermüdender Präsentation er wegen seiner Kritiker genötigt sei. Der große Zeit- und Kostenaufwand, so Reden weiter, erlaube es ihm als einzelnem Autor nun nicht mehr, seine größeren statistischen Arbeiten noch weiter fortzusetzen (Reden 1854, S. ix f.). Die ,gedrängteren“ Staatenvergleiche, die Reden hier meinte, betrafen etwa die Rohseideerzeugung (in der Welt) und den Rohseideverbrauch (in Europa) (S. 397), die Erzeugung von Roheisen und Gusseisen (S. 457), die Kohleförderung (S. 464f.), die Schiffszahl und Tonnage der Handelsflotten europäischer Staaten (S. 550f.) oder den Wert der aus den Zollregistern sich ergebenden Ein- und Ausfuhr (S. 642-646). Diese Tabellen mit den Werten für die einzelnen Staaten waren zwar nicht als Staatenranking dargeboten, erlaubten aber durch die Nennung der Prozentanteile an den jeweiligen Gesamtsummen relativ leicht die Ermittlung der Spitzenreiter und Schlusslichter, im zuletzt genannten Beispiel also den Anteil der Staaten am damaligen Welthandel, differenziert nach Ein- und Ausfuhr, und, in einer zusätzlichen Spalte, umgerechnet auf einen Wert pro Kopf der Bevölkerung. Temporalisierte Vergleiche, etwa von Wachstumsraten, waren auf der Basis der Publikation von Reden allerdings nicht möglich. Er konnte für seine höher aggregierten vergleichenden Zahlen nur auf halbwegs synchrone Momentaufnahmen zurückgreifen.

Innovativer hinsichtlich der Themen, Methoden und Darstellungsformen waren die statistischen Publikationen des französischen Mathematikers, Marineingenieurs und Politikers Charles Dupin (1784-1873). Zwei Ziele trieben ihn seit den 1820erJahren um. Im europäischen Maßstab sollte Frankreich von anderen Ländern lernen, vor allem von Großbritannien, dem vorläufigen Sieger im kommerziell-industriellen wie auch militärischen Wettbewerb (vgl. Romani 2002, S. 99-106). Und innerhalb Frankreichs sollte der Süden den von Dupin mit Zahlen belegten Rückstand zu den nördlichen Regionen des Landes aufholen. Dupins großes Werk über die Forces productives et commerciales de la France war in diesem Sinne seinen „Compatriotes du Midi“" gewidmet (Dupin 1827, Bd. 1, S. i). Zu den „Produktivkräften“, die laut Dupin besonders im Süden anzuregen seien, gehörte die Erziehung der Jugend. Kurz zuvor, am 30. November 1826, hatte Dupin in einer Rede vor dem Conservatoire des Arts et Métiers bereits eine Frankreichkarte präsentiert, die für alle französischen Départments die Zahl der Primarschüler im Verhältnis zur Bevölkerungszahl durch verschiedene Schwarz- und Grautöne anzeigte. Diese "Carte figurative de l'instruction populaire de la France" sollte den Rückstand der 54 Départments südlich einer Linie von Saint-Malo bis Genf im Vergleich zu den 32 nördlich dieser Linie gelegenen Départements veranschaulichen. Demnach schickten die 13 Mio. Bewohner des Nordens 740.846 Kinder zur Schule, die 18 Mio. Bewohner des Südens hingegen nur 375.931 Kinder (Dupin 1826, S. 27, 1827, Bd. 2, S. 251, Planche I). Dupins visuelle Darstellung des Vorsprungs oder der Zurückgebliebenheit 
von Regionen in Form von Karten wirkte schnell schulbildend (vgl. Palsky 1996, S. 59-77). Adriano Balbi und André-Michel Guerry produzierten 1829 eine Statistique comparée, in der sie drei Frankreichkarten für Schulbildung, Verbrechen gegen Personen und Verbrechen gegen das Eigentum einander gegenüberstellten; zumindest hinsichtlich der Eigentumsdelikte schnitt Frankreichs Süden dabei deutlich besser ab als der Norden (Balbi und Guerry 1829). Guerry legte dann 1833 in seinem Essai sur la statistique morale de la France weitere Karten gleicher Art vor, unter anderem zu Spenden für die Armen und zu Selbstmorden, bei letzteren erneut mit negativem (tiefschwarz gefärbtem) Ergebnis für den Norden im Vergleich zum Süden Frankreichs (Guerry 1833, Planches V, VI). Guerrys Karten zur Moralstatistik waren mithin geeignet, Dupins Ergebnisse zum Vorsprung des Nordens bei Schulbildung und anderen Produktivkräften teilweise zu relativieren.

Innovativ waren Dupins Berechnungen der „Produktivkräfte“ von Regionen und Staaten auch insofern, als er früher übliche Indikatoren wie Flächengröße, Bevölkerungszahl und-dichte, Steueraufkommen oder bloße Summen oder Geldwerte landwirtschaftlicher und industrieller Produktion zurückwies (Dupin 1827, Bd. 1, S. 1-9). Stattdessen setzte er die Arbeitskraft eines männlichen Arbeiters im Alter zwischen 17 und 54 Jahren als Maßeinheit (travailleur effectif) und berechnete danach die Gesamtkraft aller menschlichen Arbeitenden, Tiere (Pferde, Rinder, Esel) und Maschinen (Wasser- und Windmühlen, Schiffssegel, Dampfmaschinen) eines Landes, differenziert nach landwirtschaftlichem und industriellem Sektor. Die ungleich höhere Produktivkraft des Vereinigten Königreichs (Großbritannien und Irland) im Vergleich zu Frankreich ergab sich danach vor allem aus dem Maschineneinsatz in der Industrie (Dupin 1827, Bd. 1, S. 19-29). Beunruhigender aus französischer Sicht war Dupins Berechnung der jährlichen Wachstumsraten beider Staaten von 1780-1826 in dieser Maßeinheit; das britische jährliche Wachstum an „travailleurs effectifs“ lag demnach in diesem Zeitraum fast dreimal so hoch wie das französische (628.010 zu 217.092) (Dupin 1827, Bd. 1, S. 31).

Verglichen mit den vorwiegend noch auf absoluten Summen- und Größenangaben basierenden Staatenvergleichen in den Werken Bisingers oder von Redens waren die innerfranzösischen Nord-Süd-Vergleiche und bilateralen französisch-britischen Vergleiche im Stile Dupins thematisch weiter gefasst (Bildung, Moral), methodisch avancierter (Produktivkräfte-Berechnung) und darstellungstechnisch raffinierter (Karten). Für ein multilaterales (europäisches) Staatenranking, das derartigen Qualitätsmaßstäben genügt hätte, fehlten jedoch im Europa des 19. Jahrhunderts die organisatorischen Voraussetzungen: eine gleichmäßige serielle Datenerhebung in vielen Ländern und ein anerkanntes centre of calculation. Einzelne Autoren wären mit dieser Aufgabe überfordert gewesen. Internationale, auf Schaueffekte angelegte Vergleichsinszenierungen - die Weltausstellungen - fungierten seit 1851 und bis ins beginnende 20. Jahrhundert als multimediales Dispositiv, um das Potenzial und die relative Fort- oder Rückschrittlichkeit von Staaten sichtbar werden zu lassen.

\subsection{Die Weltausstellungen (1851-1900) als Leistungs- und Zivilisationsvergleiche}

Die Weltausstellungen der zweiten Hälfte des 19. Jahrhunderts waren Indikator und treibender Faktor des Prestigewettbewerbs der Staaten vor einem nun auch Laien in 
zunehmender Zahl umfassenden Publikum. Etwa 6Mio. Besucher kamen 1851 zur ersten Weltausstellung, der Great Exhibition of the Works of Industry of all Nations im Londoner Kristallpalast; die Exposition internationale universelle von 1900 in Paris zog 48 Mio. Touristen aus aller Welt an (Brockmeier 2010, S. 25). Nur die wenigsten von ihnen dürften systematische Staatenvergleiche angestellt haben, doch in nahezu allen zeitgenössischen Pressekommentaren und Berichten von Privatleuten waren die mehr oder weniger gelungene Präsentation des eigenen Herkunftslands, sein mehr oder weniger erfolgreiches Abschneiden bei der Vergabe der Preise sowie das Überholen, Aufholen oder Zurückbleiben rivalisierender Staaten dominante Themen (Beispiele für London: Scherer 1851; für Wien: Ujvári 2011; für Paris: Mölk und Detering 2010). Die Weltausstellungen ließen das relative Prestige der Staaten in seinen verschiedenen Dimensionen (vgl. Werron 2012) anschaulich werden: Sie zeigten spezifische Leistungen in einzelnen Bereichen (Kunst, Technik, Bildung, Hygiene) oder Produktlinien (Maschinen, Agrarprodukte) ebenso wie das allgemeine Modernitätsgefälle zwischen den Staaten. Das von den individuellen Ausstellern und den vor- und nachbereitenden nationalen Komitees sorgsam registrierte Ausmaß der Aufmerksamkeit des Publikums war ein Indiz für Erfolg. Die durch die Jury vergebenen Preise (Gold-, Silber- und Bronzemedaillen) wurden national zugerechnet und wie der „Medaillenspiegel“ bei den Olympischen Spielen seit 1896 als Leistungsvergleich gelesen. Die Zahl und Bedeutung der hochrangigen Besucher, vor allem der Staatsoberhäupter, verschaffte den Regierenden der Gastgeberländer zusätzliches Gewicht und Legitimität vor den Augen der eigenen wie der europäischen und globalen Öffentlichkeit. Letzteres galt vor allem für Frankreich, das nicht zuletzt deshalb zum Schauplatz der meisten Weltausstellungen des 19. Jahrhunderts wurde, weil die je neuen Machtinhaber sie gezielt als Legitimitätsbeweis einzusetzen suchten: mit beachtlichem Erfolg im Fall Napoleons III., der 1855 in Paris durch den Besuch Königin Victorias und 1867 durch die Besuche des Zaren, des preußischen Königs und Bismarcks geehrt wurde; mit deutlich weniger Erfolg im Fall der frühen Dritten Republik, da die Pariser Ausstellung von 1878 vom Deutschen Reich und die durch den Eiffelturm berühmt gewordene Ausstellung von 1889 wegen ihres symbolischen Datums (Jahrhundertfeier der Französischen Revolution) sogar von mehreren europäischen Monarchien (Österreich-Ungarn, Russland, Deutschland und Großbritannien) boykottiert wurde. Einen Legitimitätszuwachs verzeichnete die Dritte Republik hingegen bei der Ausstellung von 1900, die das Ende der außenpolitischen Isolation Frankreichs unter anderem durch die Einweihung des Pont Alexandre III anzeigte, zu dem zuvor Zar Nikolaus II. 1896 den Grundstein gelegt hatte (Haltern 1973, S. 5-11).

$\mathrm{Zu}$ den Topoi der öffentlichen Begleitrhetorik der Ausstellungen gehörte in den 1850er- und 1860er-Jahren der Verweis auf den friedlichen Wettbewerb, der die kriegerischen Konflikte der Vergangenheit abgelöst und die Rivalität stattdessen auf die Felder der Industrie, des Handels, der Künste und Wissenschaften, mit einem Wort: der Zivilisation, gelenkt habe. Die Weltausstellungen förderten mithin die Verlagerung der Staatenvergleiche von der Macht zu Leistung und Kultur. Sie erzeugten, so schrieb die Times im Oktober 1849, eine neue Form der Rivalität: „the rivalry of civilized art, instead of the old rivalry of strategic skill and brute force“" (zit. nach Haltern 1971, S. 51). Eine Vorliebe für martialisches Vokabular blieb indes Teil 
der Rhetorik des friedlichen Wettbewerbs, besonders im Selbstvergleich der Franzosen mit Großbritannien. Kein anderer als Charles Dupin, nun in seiner Eigenschaft als Präsident der französischen Kommission zur Vorbereitung der Londoner Ausstellung, richtete im Juli 1850 vor der Abgeordnetenkammer seinen Appell an die französischen Aussteller, das Vaterland auf diesem ,Schlachtfeld des Friedens“ genauso würdig zu vertreten, wie es die „Väter in den Tagen unserer Triumphe auf dem Schlachtfeld des Krieges“" getan hätten. Und im Juni 1851 berichtete Adolphe Thiers in einer Parlamentsrede zur Verteidigung des französischen protektionistischen Systems von der „größten patriotischen Freude“ seines Lebens, die er während seines Besuchs der Londoner Ausstellung empfunden habe. Vor 40 Jahren sei es die Armee gewesen, die Frankreich mit Ruhm bedeckt habe, nun sei es seine Industrie, die es „,vor den Augen Europas“ ehre und ihn überzeugt sein lasse, dass Frankreich nach wie vor „die intelligenteste der Nationen“ sei (Zitate nach Haltern 1971, S. 230, 232 f., meine Übers.).

Etwas andere Akzente setzte auf britischer Seite der Gemahl Königin Victorias, Prinz Albert, auf dessen Betreiben die Londoner Ausstellung wesentlich zustande kam. In seiner Rede im Mansion House anlässlich eines Banketts des Lord Mayor of London warb er im März 1850 für die Ausstellung mit der Aussicht, dass sie die Gegenwart dem Ziel aller Geschichte näherbringe, der „unity of mankind“. Nicht einer Einheit allerdings, so präzisierte er, ,which breaks down the limits and levels the peculiar characteristics of the different nations of the earth, but rather a unity, the result and product of those very national varieties and antagonistic qualities" (zit. nach Haltern 1971, S. 102). Neben dem kompetitiven Nationenvergleich (,,besserschlechter") auf einer temporalen Skala des Fortschritts, den Prinz Albert durchaus auch erwähnte, lenkte er hier den Blick auf eine andere Vergleichsoption: die Betonung der Eigenart, Besonderheit oder sogar Einmaligkeit der Vergleichseinheiten, hier also der Nationen (,einfach anders“) (vgl. Steinmetz 2019a).

Beide Varianten des Staatenvergleichs, die temporalisierten Besser-schlechterVergleiche und die synchronen Einfach-anders-Vergleiche waren auf den Weltausstellungen möglich und wurden dort visuell und rhetorisch bedient, letztere häufig von Ausstellern und Staaten, die im Wettbewerb um die Spitzenplätze industrielltechnischer Produktion offenkundig nicht mithalten, hingegen in Feldern wie der Kunst, Architektur oder Lebensart Achtungserfolge erzielen konnten. Beide Vergleichsvarianten wurden in London 1851 im Übrigen nicht nur auf europäische Staaten (,Nationen“) und ihre Imperien, sondern auch mit Blick auf andere kollektive Einheiten angewandt: die „Rassen“ innerhalb Europas (lateinische, germanische, keltische „Rassen“) sowie die großen christlichen Konfessionen (protestantisches und katholisches Europa). Es entstand eine Art Chrono-Topografie der relativen Fortschritte wie auch der jeweiligen Eigenarten. Sie blieb in London 1851 noch weitgehend auf Europa beschränkt (vgl. Young 2009, bes. S. 107-123), erfasste bei den Ausstellungen seit den 1870er-Jahren aber zunehmend die Staaten, „Rassen“ und Religionsgemeinschaften der ganzen Welt, im 20. Jahrhundert dann auch die politischen Systeme und Ideologien, besonders markant bei der Pariser Ausstellung von 1937.

Welche Vergleiche unvoreingenommene Besucher vollziehen konnten, wurde wesentlich durch die jeweilige Klassifikation der ausgestellten Produkte oder Errun- 
genschaften und durch deren räumliche Ordnung, also die Architektur des Ausstellungsgeländes, vorstrukturiert. Im Prinzip denkbar waren einerseits räumliche Anordnungen nach bestimmten Produktklassen, Fabrikationsprozessen (vom Rohstoff zum Fertigprodukt) oder Errungenschaften (Hygiene, Arbeiterwohnungen), andererseits Einteilungen nach Staaten (,Nationen“) und deren Imperien, die ihrerseits zu größeren Staatengruppen (deutsche Staaten des Zollvereins, Weltregionen) zusammengefasst sein konnten. In London 1851 wie auch bei den meisten Folgeausstellungen bis ins 20. Jahrhundert herrschte bei der Raumgestaltung das Staaten- oder Imperienprinzip vor, wobei für die Gastgebernation stets die größte Ausstellungsfläche und ein prominent platzierter Raum des Geländes reserviert waren. Das relative Gewicht der Staaten wurde auf diese Weise gut sichtbar gemacht, doch entstand der Nachteil, dass Leistungsvergleiche der Staaten in spezifischen Feldern erschwert wurden.

Die weitaus originellste Lösung des Problems, mehrere Arten von Vergleichen zu ermöglichen, gelang durch das vom Sozialreformer Frédéric Le Play entworfene Hauptgebäude der Pariser Weltausstellung von 1867 (vgl. Hanninger 1867; Barth 2007, bes. S. 161-165; Steinmetz 2019b, S. 367 ff.). Es handelte sich um ein riesiges Oval von 500 Metern Durchmesser in der Länge und 386 Metern in der Breite, dessen Gesamtfläche nach den ausstellenden Staaten in Sektionen, also mehr oder weniger große „Tortenstücke“, aufgeteilt war. Wenn die Besucher die konzentrischen Ringstraßen des Ovals durchwanderten, konnten sie jeweils die Erzeugnisse einer Klasse (z. B. Maschinen im äußersten Ring) betrachten und im Laufe ihres Rundgangs die Leistungen der ausstellenden Staaten auf diesem Feld miteinander vergleichen. Die relative Fort- oder Rückschrittlichkeit wurde so erlebbar. Ging man hingegen auf den Wegen von außen nach innen zum ,zentralen Garten“, bewegte man sich innerhalb eines Staats oder einer Staatengruppe von Produktklasse zu Produktklasse und gewann so einen Eindruck von der Gesamtleistung des jeweiligen Staats. So wurde dann dessen eigenartiges Profil erkennbar (Abb. 2).

Der Bereich außerhalb der ovalen Haupthalle war als Landschaftspark mit Pavillons gestaltet, die teils besonderen nichtindustriellen Bereichen (Landwirtschaft und Tiere, Arbeitersiedlungen), teils landestypischer Architektur (norwegisches Chalet, russisches Dorf, chinesischer Kiosk) gewidmet waren und so nochmals die Eigenart - auch Exotik - einzelner „Nationen“ vor Augen führten.

Gegen Ende des 19. Jahrhunderts, beginnend mit der Ausstellung in Wien 1873, mehrten sich in Privatbriefen und Presseartikeln die Klagen über eine allgemeine „Ausstellungsmüdigkeit“ (Brockmeier 2010; Geppert 2002, S. 39 f.). Mit dem wachsenden Publikumsandrang, so die Kritiker, sei die Massenunterhaltung an die Stelle der ernsthaften Produkt- und Leistungsvergleiche getreten. Eine (national-)staatliche oder imperiale Prestigeangelegenheit blieb der Wettbewerb um Preise und Aufmerksamkeit jedoch weiterhin. In einer Zeit, als daten- und zahlenbasierte, europaweite oder gar globale Staatenvergleiche mangels einer statistischen Infrastruktur und wegen fehlender Einigkeit über Kategorien, Zähl- und Rechenmethoden nicht möglich waren, boten die Weltausstellungen eine Möglichkeit, auf visueller Anschauung beruhende Staatenrankings als Momentaufnahmen zu vollziehen. Diese waren freilich in ihrer Kriterienwahl und -anwendung zu keiner Zeit stabil und konnten insofern auch keine Serien bilden. 


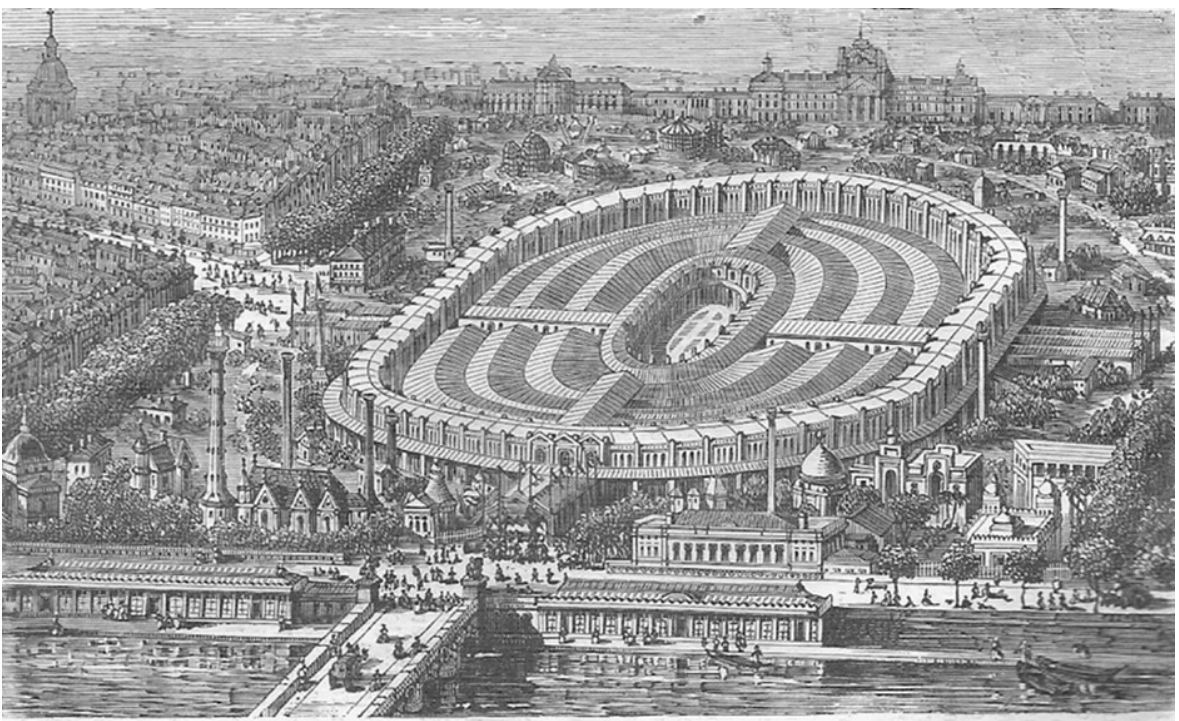

Palais de l'Exposition universelle de 1867, vu à vol d'oiseau.

Abb. 2 Stich aus: Alfred Joanne, Paris-Diamant. Collection des guides Joanne, Paris: Hachette, 1867, https://de.wikipedia.org/wiki/Datei:Exposition_universelle_de_1867.png

\section{Fazit und Ausblick: International-vergleichende Statistik zwischen Wunsch und Realität}

Praktiken des Staatenvergleichs und Versuche, Staaten unter bestimmten Gesichtspunkten in eine Rangordnung zu bringen, haben eine lange Tradition. Die zahlenbasierte ,,politische Arithmetik“ und die zunächst verbal-beschreibende, später auch rechnende „Statistik“ stellten die Staatenvergleiche auf eine neue Grundlage. Seit dem späten 17. Jahrhundert waren europäische Gelehrte und Staatsdiener von der Idee fasziniert, ihre Vergleiche und Hierarchisierungen von Staaten durch seriell zu erhebende Kennzahlen, aber auch durch qualitative Verfahren der Landesaufnahme so zu gestalten, dass die temporale Dimension einbezogen werden konnte, also relative Verbesserungen oder Verschlechterungen über längere Zeiträume hinweg erfassbar würden. In einem Zeitalter mehr oder weniger permanenter Kriege von 1688-1815 standen dabei zunächst die Messung, Zählung und Berechnung von Machtressourcen (,Staatskräften“), also Flächengrößen, Bevölkerungszahlen, Bodenerträgen, Staatsfinanzen und Land- und Seestreitkräften im Zentrum. Mit der Bevölkerungsdichte gelangte dann um 1780 bereits ein Indikator für die relative Kulturhöhe eines Staates ins Spiel. Damit begann ein im 19. Jahrhundert sich verstärkender Prozess, in dessen Verlauf sich das Vergleichsinteresse der Statistiker von der reinen Macht über Leistungen materieller wie immaterieller Art bis hin zum Zivilisierungsgrad eines Staates ausdehnte. Zunehmend raffinerter gestaltete Tabellen, grafische Darstellungen und Karten dienten den Statistikern zur vereinfachenden Veranschaulichung ihrer Befunde. 
Die Praktiker des statistischen Staatenvergleichs seit dem späten 17. Jahrhundert nahmen als Handlungsroutine vorweg, was die Spätaufklärer und Idealisten des Revolutionszeitalters um 1800 explizit als progressive Geschichtsphilosophie formulierten, deren empirischer Kern letztlich stets darin bestand, dass miteinander rivalisierende Staaten, „Nationen“ oder Erdteile (Europa) - bei Karl Marx kamen die „Klassen“ und im späteren 19. Jahrhundert die „Rassen“ hinzu - als gedachte Subjekte den Fortschritt der gesamten Menschheit herbeiführten. Gegen diese Geschichtsauffassung wie auch gegen die den vermeintlichen Fortschritt auf wenige Zahlenreihen reduzierenden Tabellen der Statistiker regte sich etwa seit 1800 vermehrt Widerspruch. Diese Kritik trat im Namen des Eigentümlichen, Besonderen und Singulären auf. Nicht gegen das Vergleichen als solches richtete sich die Kritik, auch nicht grundsätzlich gegen Zählungen und Berechnungen, sondern gegen eine Art des Vergleichens, die wesenhafte Unterschiede oder Einmaligkeiten durch Rubrizierung unter wenige Kategorien einebnete, um die verglichenen Entitäten, in diesem Fall Staaten, auf einer einfachen Rangordnung von „,besser“ oder „schlechter“ verorten zu können. Das Ergebnis von Vergleichen müsse auch sein können, dass die verglichenen Einheiten ,einfach anders“ seien. Seit dem Beginn des 19. Jahrhunderts begleitet diese Kritik alle Versuche des Rankings von Staaten oder anderen Entitäten.

Nicht so sehr mit diesem theoretischen Einwand, sondern eher mit den praktischen Herausforderungen der Datengewinnung und ihrer Verarbeitung hatten diejenigen Autoren zu kämpfen, die sich im 18. und 19. Jahrhundert an die mühevolle Arbeit zahlen- und datenbasierter Staatenvergleiche heranwagten. Auch der allmähliche Aufbau einzelstaatlicher statistischer Bureaus seit dem frühen 19. Jahrhundert änderte daran nur wenig. Zum Teil ließ er die Aufgabe des Staatenvergleichs sogar noch schwieriger werden, weil nun zum einen immer mehr Daten aus den Einzelstaaten zur Verfügung standen, zum anderen deren uneinheitliche Kategorisierung zu einem immer offenkundigeren Problem wurde. In der zweiten Hälfte des 19. Jahrhunderts wurde es auf den internationalen statistischen Kongressen der Experten zwar diskutiert, aber bis zum Ersten Weltkrieg nicht gelöst, während im gleichen Zeitraum das öffentliche Interesse an kompetitiven Staatenvergleichen durch die Nationen- und Imperienkonkurrenz erneut stark anwuchs. Die Weltausstellungen seit 1851 boten einen gewissen Ersatz für das Publikumsinteresse an Staatenvergleichen, befriedigten aber nicht die Ansprüche der Expertengruppen und Bürokratien, die für ihre Bewertungen und darauf gegründete Entscheidungen verlässliches, und das hieß statistisches Wissen forderten.

Ohne eine anerkannte zentralisierte Instanz, ein centre of calculation, war dieses Postulat nicht einzulösen. Die Unterorganisationen des 1919 geschaffenen Völkerbunds schufen dafür in Teilbereichen erste Voraussetzungen, darunter eine Economic and Financial Organization für die Wirtschaftsstatistik (vgl. Bemmann 2019b) und die International Labour Organization (ILO) mit weiteren Unterorganisationen für die Sozial- und Arbeiterstatistik. Sie sahen ihre Hauptaufgabe zunächst darin, auf eine Standardisierung der Kategorien hinzuwirken, nach denen in den Einzelstaaten die Daten erhoben und die Statistiken erstellt wurden, sodass diese dann - im zweiten Schritt - zur Basis einer zukünftigen vergleichenden Staatenbeobachtung und Staatenbewertung hinsichtlich der Einhaltung bestimmter Standards, etwa der 
Umsetzung von Arbeitszeitnormen, werden konnten. Kategorien wie „Erwerbstätigkeit" (gainfully occupied population) wurden auf diesem Weg einer internationalen Vereinheitlichung näher gebracht und damit für Staatenvergleiche tauglich gemacht (vgl. Wobbe 2019). Insoweit vermochten die Unterorganisationen des Völkerbunds, allen voran die ILO, tatsächlich als ,standardsetzende Agenturen“ zu wirken (Kott und Droux 2013, S. 4). Von den heutigen Praktiken des Staatenrankings war man in der Zwischenkriegszeit gleichwohl noch einige Schritte entfernt. Denn einer breiteren Öffentlichkeit, selbst innerhalb der Politik, blieb die Arbeit der ILO und anderer Unterorganisationen des Völkerbunds weitgehend verborgen. Die Experten blieben unter sich, und so konnte jener Mechanismus des shame and blame vor der Weltöffentlichkeit nicht greifen, dem heutige Staatenrankings allererst ihre Wirksamkeit in dem Sinne verdanken, dass sich die Staatsregierungen entweder den im Ranking implizierten Normerwartungen fügen (compliance) oder aber durch das Propagieren neuer Kategorien und Bewertungskriterien ein anderes Ranking zu etablieren suchen - und damit erneut das Prinzip der hierarchisierenden Staatenbewertung fortschreiben.

Danksagung Der Beitrag entstand im Rahmen des von der Deutschen Forschungsgemeinschaft geförderten Bielefelder Sonderforschungsbereichs (SFB) 1288 „Praktiken des Vergleichens. Die Welt ordnen und verändern" (TP C03: Historische Semantik des Vergleichens, 16.-20. Jahrhundert). Für eine kritische Lektüre des Manuskripts und wertvolle Hinweise danke ich Jürgen Osterhammel.

Funding Open Access funding enabled and organized by Projekt DEAL.

Open Access Dieser Artikel wird unter der Creative Commons Namensnennung 4.0 International Lizenz veröffentlicht, welche die Nutzung, Vervielfältigung, Bearbeitung, Verbreitung und Wiedergabe in jeglichem Medium und Format erlaubt, sofern Sie den/die ursprünglichen Autor(en) und die Quelle ordnungsgemäß nennen, einen Link zur Creative Commons Lizenz beifügen und angeben, ob Änderungen vorgenommen wurden.

Die in diesem Artikel enthaltenen Bilder und sonstiges Drittmaterial unterliegen ebenfalls der genannten Creative Commons Lizenz, sofern sich aus der Abbildungslegende nichts anderes ergibt. Sofern das betreffende Material nicht unter der genannten Creative Commons Lizenz steht und die betreffende Handlung nicht nach gesetzlichen Vorschriften erlaubt ist, ist für die oben aufgeführten Weiterverwendungen des Materials die Einwilligung des jeweiligen Rechteinhabers einzuholen.

Weitere Details zur Lizenz entnehmen Sie bitte der Lizenzinformation auf http://creativecommons.org/ licenses/by/4.0/deed.de.

\section{Literatur}

Achenwall, Gottfried. 1749. Abriß der neuesten Staatswissenschaft der vornehmsten Europäischen Reiche und Republicken zum Gebrauch in seinen Vorlesungen. Göttingen: Schmidt.

Balbi, Adriano, und André-Michel Guerry. 1829. Statistique comparée de l'état de l'instruction et du nombre des crimes dans les divers arrondissements des académies et des cours royales de France. Paris: Jules Renouard.

Barth, Volker. 2007. Mensch versus Welt. Die Pariser Weltausstellung von 1867. Darmstadt: Wissenschaftliche Buchgesellschaft.

Behrisch, Lars. 2016a. Die Berechnung der Glückseligkeit. Statistik und Politik in Deutschland und Frankreich im späten Ancien Régime. Ostfildern: Thorbecke Verlag.

Behrisch, Lars. 2016b. Statistics and Politics in the $18^{\text {th }}$ Century. In Conventions and Quantification Transdisciplinary Perspectives on Statistics and Classifications, Special Issue: Historical Social Research 41/2. Hrsg. Rainer Diaz-Bone und Emmanuel Didier, 238-257. 
Bemmann, Martin. 2019a. Comparing Economic Activities on a Global Level in the 1920s and 1930s. Motives and Consequences. In The Force of Comparison. A New Perspective on Modern European History and the Contemporary World, Hrsg. Willibald Steinmetz, 242-265. New York/Oxford: Berghahn Books.

Bemmann, Martin. 2019b. Internationale Weltwirtschaftsstatistik. Beobachtungen, Überlegungen und Thesen zur Genese internationaler Wirtschaftsstatistik in den 1920er und 1930er Jahren. In Die Zählung der Welt. Kulturgeschichte der Statistik vom 18. bis 20. Jahrhundert, Hrsg. Stefan Haas, Michael C. Schneider und Nicolas Bilo, 195-217. Stuttgart: Franz Steiner Verlag.

Bertuch, Carl. [1814/15] 1916. Carl Bertuchs Tagebuch vom Wiener Kongreß. Hrsg. Hermann Freiherr von Egloffstein. Berlin: Verlag Gebr. Paetel.

Bhuta, Nehal. 2015. Measuring Stateness, Ranking Political Orders. Indices of State Fragility and State Failure. In Ranking the World. Grading States as a Tool of Governance, Hrsg. Alexander Cooley und Jack Snyder, 85-111. Cambridge: Cambridge University Press.

Bisinger, Joseph Constantin. 1823. Vergleichende Darstellung der Grundmacht oder der Staatskräfte aller europäischen Monarchien und Republiken. Pest und Wien: C.A. Hartleben.

Brockmeier, Alke. 2010. Die Pariser Weltausstellung in deutschen Kulturzeitschriften. In Perspektiven der Modernisierung. Die Pariser Weltausstellung, die Arbeiterbewegung, das koloniale China in europäischen und amerikanischen Kulturzeitschriften um 1900, Hrsg. Ulrich Mölk und Heinrich Detering, 25-40. Berlin/New York: de Gruyter.

Burke, Edmund. [1790] 1989. The Writings and Speeches of Edmund Burke. Vol. VIII: The French Revolution 1790-1794. Hrsg. L. G. Mitchell und William B. Todd. Oxford: Clarendon Press.

Chalmers, George. 1804. An Estimate of the Comparative Strength of Great Britain. London: John Stockdale.

Chalmers, Georg. 1786. Schätzung der verhältnißmäßigen Stärke von Großbritannien. Aus dem Englischen von Valentin August Heinze. Berlin: Friedrich Nicolai.

Cooley, Alexander, und Jack Snyder (Hrsg.). 2015. Ranking the World. Grading States as a Tool of Governance. Cambridge: Cambridge University Press.

Crome, August F. W. 1785. Über die Größe und Bevölkerung der sämtlichen europäischen Staaten. Leipzig: Weygandsche Buchhandlung.

Cullen, M. J. 1975. The Statistical Movement in Early Victorian Britain. The Foundations of Empirical Social Research. Hassocks: Harvester Press.

Damien, Robert. 2003. Prolégomènes français à une science politique future: Vauban, Lavoisier, Volney, Neufchâteau, Chaptal. In Arithmétique politique dans la France du XVIIIe siècle, Hrsg. Thierry Martin, 17-34. Paris: Institut National d'Études Démographiques.

Desrosières, Alain. 1998. The Politics of Large Numbers. A History of Statistical Reasoning. Cambridge, Mass.: Harvard University Press.

Dupin, Charles. 1826. Effets de l'enseignement populaire sur les prospérités de la France. Discours prononcé ... au Conservatoire des Arts et Métiers. Paris: Bachelier Libraire.

Dupin, Charles. 1827. Forces productives et commerciales de la France. 2 Bde. Paris: Bachelier Libraire.

Eiden-Offe, Patrick. 2015. „Oppositionelle Statistik“. Von den unterschiedlichen politischen Gebrauchsweisen statistischen Wissens im Vormärz. In Berechnen / Beschreiben. Praktiken statistischen (Nicht-) Wissens 1750-1850. Hrsg. Gunhild Berg et al., 171-192. Berlin: Duncker \& Humblot.

Gabriëls, Jos. 2017. Cutting the Cake. The Congress of Vienna in British, German and French Political Caricature. European Review of History / Revue européenne d'histoire 24:131-157.

Gatterer, Johann Christoph. 1773. Ideal einer allgemeinen Weltstatistik. Göttingen: Vandenhökischer Verlag.

Geppert, Alexander C. T. 2002. Welttheater. Die Geschichte des europäischen Ausstellungswesens im 19. und 20. Jahrhundert. Ein Forschungsbericht. Neue politische Literatur 47:10-61.

Geppert, Alexander C. T. 2013. Weltausstellungen. In Europäische Geschichte Online (EGO), Hrsg. Leibniz-Institut für Europäische Geschichte (IEG), Mainz 2013-06-20, http://www.ieg-ego.eu/gepperta2013-de. (Zugegriffen: 16 Feb 2021).

Gierl, Martin. 2015. Johann Christoph Gatterers Ideal einer allgemeinen Weltstatistik. Die praktische Aufklärung fortlaufender Geschichte und ihre Wissensarchitektur. In Berechnen / Beschreiben. Praktiken statistischen (Nicht-) Wissens 1750-1850, Hrsg. Gunhild Berg et al., 21-41. Berlin: Duncker \& Humblot.

Glass, D. V. 1973. Numbering the people. The eighteenth-century population controversy and the development of census and vital statistics in Britain. Farnborough: Saxon House.

Göderle, Wolfgang. 2018. Das Wissen des modernen Staates. Standardisierung und Internationalisierung als externe Einflussfaktoren in der staatlichen Wissenserzeugung. In Dynamiken der Wissensproduk- 
tion. Räume, Zeiten und Akteure im 19. und 20. Jahrhundert, Hrsg. Wolfgang Göderle und Manfred Pfaffenthaler, 175-206. Bielefeld: Transcript Verlag.

Graunt, John. 1662. Natural and Political Observations ... upon the Bills of Mortality. London: Tho. Roycroft.

Guerry, André-Michel. 1833. Essai sur la statistique morale de la France. Paris: Crochard.

Haltern, Utz. 1971. Die Londoner Weltausstellung von 1851. Ein Beitrag zur Geschichte der bürgerlichindustriellen Gesellschaft im 19. Jahrhundert. Münster: Verlag Aschendorff.

Haltern, Utz. 1973. Die „Welt als Schaustellung“. Zur Funktion und Bedeutung der internationalen Industrieausstellung im 19. und 20. Jahrhundert. Vierteljahrschrift für Sozial- und Wirtschaftsgeschichte $60: 1-40$

Hanninger, Anton. 1867. Das Ausstellungsgebäude für 1867 in Paris. Allgemeine Bauzeitung (Wien) 32:112-136 u. 18-36 (Pläne).

Hassel, [Johann] G[eorg] Heinrich. 1809. Statistische Uebersichts-Tabellen der sämmtlichen Europäischen und einiger aussereuropäischen Staaten. Göttingen: Dieterichsche Buchhandlung.

Heintz, Bettina. 2012. Welterzeugung durch Zahlen. Modelle politischer Differenzierung in internationalen Statistiken, 1948-2010. Soziale Systeme 18:7-39.

Hirschi, Caspar. 2005. Wettkampf der Nationen. Konstruktionen einer deutschen Ehrgemeinschaft an der Wende vom Mittelalter zur Neuzeit. Göttingen: Wallstein Verlag.

Hont, István. 2010. Jealousy of Trade. International Competition and the Nation-State in Historical Perspective. Cambridge Mass.: Belknap Press.

Innes, Joanna. 2009. Inferior politics: social problems and social policies in eighteenth-century Britain. Oxford: Oxford University Press.

King, Gregory. [1696]. The Earliest Classics: John Graunt, Natural and Political Observations made upon the Bills of Mortality (1662); Gregory King, Natural and Political Observations and Conclusions upon the State and Condition of England, 1696 (1804). Hrsg. Peter Laslett, 29-69. Westmead, Farnborough: O. Gregg International Publishers Limited 1973.

Klüber, Johann Ludwig. 1815. Acten des Wiener Congresses in den Jahren 1814 und 1815. Bd. 5. Erlangen: Palm und Enke (ND Osnabrück: Otto Zeller, 1966), 1-120 (Acten der statistischen Commission auf dem Wiener Congreß).

Klueting, Harm. 1986. Die Lehre von der Macht der Staaten. Das außenpolitische Machtproblem in der „politischen Wissenschaft" und in der praktischen Politik im 18. Jahrhundert. Berlin: Duncker \& Humblot.

Koselleck, Reinhart. 1979. Vergangene Zukunft. Zur Semantik geschichtlicher Zeiten, Frankfurt a. M.: Suhrkamp Verlag.

Kott, Sandrine, und Joëlle Droux. 2013. Introduction: A Global History Written from the ILO. In Globalizing Social Rights. The International Labour Organization and Beyond. Hrsg. Sandrine Kott und Joëlle Droux, 1-14. Houndsmills: Palgrave Macmillan.

Leibniz, Gottfried Wilhelm. [1680a] 1986. Entwurff gewisser Staats-Tafeln. In Sämtliche Werke. Vierte Reihe: Politische Schriften, Bd. 3: 1677-1689. Hrsg. Akademie der Wissenschaften der DDR, 340-349. Berlin: Akademie-Verlag.

Leibniz, Gottfried Wilhelm. [1680b] 1986. Bestellung eines Registratur-Amts. In Sämtliche Werke. Vierte Reihe: Politische Schriften, Bd. 3: 1677-1689. Hrsg. Akademie der Wissenschaften der DDR, 376-381. Berlin: Akademie-Verlag.

Leonhard, Jörn, und Ulrike von Hirschhausen. 2009. Empires und Nationalstaaten im 19. Jahrhundert. Göttingen: Vandenhoeck \& Ruprecht.

Mölk, Ulrich, und Heinrich Detering (Hrsg.). 2010. Perspektiven der Modernisierung. Die Pariser Weltausstellung, die Arbeiterbewegung, das koloniale China in europäischen und amerikanischen Kulturzeitschriften um 1900. Berlin/New York: de Gruyter.

Moses, Julia. 2019. Comparison and the Welfare State in Modern Europe, c. 1880-1945. In The Force of Comparison: A New Perspective on Modern European History and the Contemporary World, Hrsg. Willibald Steinmetz, 191-213. New York/Oxford: Berghahn Books.

Nikolow, Sybilla. 2001. A.F.W. Crome's Measurements of the „Strength of the State“: Statistical Representations in Central Europe around 1800. History of Political Economy 33 (Supplement):23-56.

Nipperdey, Justus. 2015. Ehre durch Zahlen. Publizistische Rangstreitigkeiten und die Evidenz der Zahl im 18. Jahrhundert. In Berechnen / Beschreiben. Praktiken statistischen (Nicht-) Wissens 1750-1850, Hrsg. Gunhild Berg et al., 43-59. Berlin: Duncker \& Humblot.

Osterhammel, Jürgen. 2009. Die Verwandlung der Welt. Eine Geschichte des 19. Jahrhunderts. München: C. H. Beck. 
Palsky, Gilles. 1996. Des chiffres et des cartes. Naissance et développement de la cartographie quantitative française au XIXe siècle. Paris: Comité des travaux historiques et scientifiques.

Pedersen, Susan. 2017. Empires, States and the League of Nations. In Internationalisms: A Twentieth-Century History, Hrsg. Patricia Clavin und Glenda Sluga, 113-138. Cambridge: Cambridge University Press.

Randeraad, Nico. 2010. States and Statistics in the Nineteenth Century. Europe by Numbers. Manchester/New York: Manchester University Press.

Reden, Friedrich Wilhelm von. 1848. Vergleichende Kultur-Statistik der Gebiets- und Bevölkerungsverhältnisse der Gross-Staaten Europa's. Berlin: Duncker.

Reden, Friedrich Wilhelm von. 1854. Deutschland und das uebrige Europa. Handbuch der Bodens-, Bevölkerungs-, Erwerbs- und Verkehrs-Statistik; des Staatshaushalts und der Streitmacht. In vergleichender Darstellung. Wiesbaden: Kreidel \& Niedner.

Reinert, Sophus A. 2014. Rivalry: Greatness in Early Modern Political Economy. In Mercantilism Reimagined: Political Economy in Early Modern Britain and Its Empire, Hrsg. Philip J. Stern und Carl Wennerlind, 348-370. Oxford: Oxford University Press.

Remer, Julius August. 1786. Lehrbuch der Staatskunde der vornehmsten europäischen Staaten. Braunschweig: Verlag der Fürstl. Waysenhausbuchhandlung.

Romani, Roberto. 2002. National Character and Public Spirit in Britain and France, 1750-1914. Cambridge: Cambridge University Press.

Rommelse, Gijs, und Roger Downing. 2014. Anglo-Dutch Mercantile Rivalry, 1585-1688. Interests, Ideologies, and Perceptions. In Merkantilismus. Wiederaufnahme einer Debatte, Hrsg. Moritz Isenmann, 169-195. Stuttgart: Franz Steiner Verlag.

Rusnok, Andrea A. 2002. Vital Accounts. Quantifying Health and Population in Eighteenth-Century England and France. Cambridge: Cambridge University Press.

Scherer, Hermann. 1851. Londoner Briefe über die Weltausstellung. Leipzig: Hermann Schultze.

Scheu, Johannes. 2015. Wider den homme moyen. Zur Soziologie des Einzelfalls. In Berechnen / Beschreiben. Praktiken statistischen (Nicht-) Wissens 1750-1850, Hrsg. Gunhild Berg et al., 193-211. Berlin: Duncker \& Humblot.

Schlegel, Friedrich. [1820-1823] 1966. Signatur des Zeitalters. In Friedrich Schlegel. Studien zur Geschichte und Politik. Hrsg. Ernst Behler. München: Verlag Ferdinand Schöningh.

Schlözer, August Ludwig. 1804. Theorie der Statistik. Nebst Ideen über das Studium der Politik überhaupt. Göttingen: Vandenhock \& Ruprecht.

Schlözer, August Ludwig. 1808. Theorie der Statistik. Göttingische Gelehrte Anzeigen 70:2089-2104.

Schulz, Wilhelm. 1831. Ueber das zeitgemäße Verhältniß der Statistik zur Politik. Morgenblatt für Gebildete Stände 25 (Beilage zu Nr. 310, 28. Dez. 1831):1-7.

Schulz, Wilhelm. 1843. Art. Staatskunde, Statistik. In Staats-Lexikon oder Encyklopädie der Staatswissenschaften, Hrsg. Carl von Rotteck und Carl-Theodor Welcker, Bd. 14, 828-838. Altona: Johann Friedrich Hammerich.

Scott, Hamish. 2009. The Fiscal-Military State and International Rivalry during the Long Eighteenth Century. In The Fiscal-Military State in Eighteenth-Century Europe. Essays in Honour of P.G.M. Dickson, Hrsg. Christopher Storrs, 23-53. Farnham: Ashgate.

Segelken, Barbara. 2010. Bilder des Staates. Kammer, Kasten und Tafel als Visualisierungen staatlicher Zusammenhänge. Berlin: Akademie-Verlag.

Slack, Paul. 2004. Measuring the National Wealth in Seventeenth-Century England. Economic History Review 57:607-635.

Slack, Paul. 2015. The Invention of Improvement. Information and Material Progress in Seventeenth-Century England. Oxford: Oxford University Press.

Speich Chassé, Daniel. 2019. In Search of a Global Centre of Calculation: The Washington Statistical Conferences of 1947. In The Force of Comparison. A New Perspective on Modern European History and the Contemporary World, Hrsg. Willibald Steinmetz, 266-287. New York/Oxford: Berghahn Books.

Speich Chassé, Daniel. 2020. Der Staatsvergleich in historischer Perspektive: Warum, seit wann und wie werden politische Mächte miteinander verglichen? In Global beobachten und vergleichen: Soziologische Analysen zur Weltgesellschaft, Hrsg. Hannah Bennani et al., 79-111. Frankfurt: Campus Verlag.

Steinmetz, Willibald. 2019a. Above/Below, Better/Worse or Simply Different? Metamorphoses of Social Comparison, 1600-1900. In The Force of Comparison. A New Perspective on Modern European History and the Contemporary World, Hrsg. Willibald Steinmetz, 80-112. New York/Oxford: Berghahn Books.

Steinmetz, Willibald. 2019b. Europa im 19. Jahrhundert. Frankfurt a. M.: Fischer Verlag. 
Twellmann, Marcus. 2015. „Ja, die Tabellen!“ Zur Heraufkunft der politischen Romantik im Gefolge numerisch informierter Bürokratie. In Berechnen /Beschreiben. Praktiken statistischen (Nicht-) Wissens 1750-1850, Hrsg. Gunhild Berg et al.,141-170. Berlin: Duncker \& Humblot.

Ujvári, Hedvig. 2011. Zwischen Bazar und Weltpolitik. Die Wiener Weltausstellung 1873 in Feuilletons von Max Nordau im Pester Lloyd. Berlin: Frank \& Timme.

Vauban, Sébastien Le Prestre de [1686]. Méthode generalle et facile pour faire le dénombrement des peuples. In Vilquin. 1975. 245-257 (Annexe).

Vilquin, Eric. 1975. Vauban, inventeur des recensements. In Annales de démographie historique. No. spéciale: Démographie historique et environnement, 207-257. Paris: Mouton. https://www.persee.fr/doc/ adh_0066-2062_1975_num_1975_1_1282. (Zugegriffen: 16 Feb 2020).

Werron, Tobias. 2012. Worum konkurrieren Nationalstaaten? Zu Begriff und Geschichte der Konkurrenz um ,weiche“ globale Güter. Zeitschrift für Soziologie 41:338-355.

Werron, Tobias. 2020. Global Publics as Catalysts of Global Competition: A Sociological View. In Global Publics. Their Power and Their Limits, 1870-1990, Hrsg. Valeska Huber und Jürgen Osterhammel, 343-366. Oxford: Oxford University Press.

Wheatley, Natasha. 2017a. Spectral Legal Personality in Interwar International Law. On New Ways of Not Being a State. Law and History Review 35:753-787.

Wheatley, Natasha. 2017b. New Subjects in International Law and Order. In Internationalisms: A Twentieth-Century History, Hrsg. Patricia Clavin und Glenda Sluga, 265-286. Cambridge: Cambridge University Press.

Wobbe, Theresa. 2019. Der internationale Wandel statistischer Repräsentationen der Arbeitswelt. Vom nationalen Zensus um 1900 zur internationalen Vergleichbarkeit in der International Labour Organization (ILO) 1882-1938. In Die Zählung der Welt. Kulturgeschichte der Statistik vom 18. bis 20. Jahrhundert, Hrsg. Stefan Haas et al., 153-171. Stuttgart: Franz Steiner Verlag.

Young, Paul. 2009. Globalization and the Great Exhibition. The Victorian New World Order, Houndsmills: Palgrave Macmillan.

Zimmermann, E[berhard] A[ugust] W[ilhelm]. 1787. A Political Survey of the Present State of Europe, in Sixteen Tables. London: C. Dilly.

Willibald Steinmetz 1957, Dr., Professor für Neuere und Neueste Geschichte, Universität Bielefeld. Forschungsgebiete: britische, deutsche und europäisch-vergleichende Geschichte politischer Begriffe und Kommunikationsformen vom 17. bis zum 20. Jahrhundert; Rechts- und Sozialgeschichte. Veröffentlichungen: Europa im 19. Jahrhundert (Neue Fischer Weltgeschichte Bd. 6). Frankfurt a. M.: S. Fischer 2019; The Force of Comparison. A New Perspective on Modern European History and the Contemporary World. Oxford 2019 (als Hrsg.). 